\title{
Debiasing the framing effect in younger and older adults' medical decision making
}

\author{
Erin L. Woodhead \\ West Virginia University
}

Follow this and additional works at: https://researchrepository.wvu.edu/etd

\section{Recommended Citation}

Woodhead, Erin L., "Debiasing the framing effect in younger and older adults' medical decision making" (2006). Graduate Theses, Dissertations, and Problem Reports. 2372.

https://researchrepository.wvu.edu/etd/2372

This Thesis is protected by copyright and/or related rights. It has been brought to you by the The Research Repository @ WVU with permission from the rights-holder(s). You are free to use this Thesis in any way that is permitted by the copyright and related rights legislation that applies to your use. For other uses you must obtain permission from the rights-holder(s) directly, unless additional rights are indicated by a Creative Commons license in the record and/ or on the work itself. This Thesis has been accepted for inclusion in WVU Graduate Theses, Dissertations, and Problem Reports collection by an authorized administrator of The Research Repository @ WVU. For more information, please contact researchrepository@mail.wvu.edu. 


\title{
Debiasing the Framing Effect in Younger and Older Adults' Medical Decision Making
}

\author{
Erin L. Woodhead
}

\author{
Thesis submitted to the \\ Eberly College of Arts and Sciences \\ at the West Virginia University \\ in partial fulfillment of the requirements \\ for the degree of
Master of Science
in
Psychology
Barry Edelstein, Ph.D., Chair Jennifer Margrett, Ph.D. B. Kent Parker, Ph.D.

\section{Department of Psychology}

Morgantown, WV

2006

Keywords: Heuristics, Framing Effect, Medical Decision Making 


\begin{abstract}
Debiasing the Framing Effect in Younger and Older Adults' Medical Decision Making Erin L. Woodhead
\end{abstract}

The framing effect is a common decisional bias. In the current study, the framing effect and its avoidance via a debiasing questionnaire was studied with younger and older adults making decisions regarding lung cancer treatment. Participants received three cancer scenarios framed in either survival or mortality terms, with scenarios presented in cumulative probability, interval probability, and life expectancy format. There were two between-subjects factors and one within-subjects factor. Younger adults exhibited the framing effect with the cumulative and interval probability formats. No framing effect was found in the debias condition. Older adults exhibited the framing effect with the interval probability format. Older adults in the debiasing condition showed the framing effect with the cumulative and interval probability formats. Older adults who had received or provided care and those who knew someone with cancer were less likely to exhibit the framing effect. Frame was the only significant predictor of treatment choice. 


\section{Acknowledgements}

There are many friends and family members I would like to thank for providing support and friendship throughout this project, and during my graduate school experience. First and foremost, I would like to thank my advisor and committee chair, Barry Edelstein, Ph.D., who was always more than willing to provide the correct amount of guidance and independence throughout this project. The knowledge I gained from him about design and implementation of research projects, as well as his dedication to helping me with my writing style, will be carried with me throughout many projects to come.

I would also like to thank the other members of my committee for providing knowledge in specialized areas. Kent Parker, Ph.D., kick started my graduate school experience with statistics, and Jennifer Margrett, Ph.D., worked closely with me on other projects that furthered my interest in research with older adults.

My family has provided invaluable support throughout all my years of school. They never fail to support me in any area that I am interested in pursuing. Their willingness to adjust their schedules in order to visit me has provided many weekends of fun adventures in West Virginia.

Many of my goals thus far would have been much more difficult to achieve had I not had such a great launch. For that, I thank Steve Zarit, Ph.D., and his lab at Penn State. Steve and his graduate students encouraged me to continue with my education, gave me invaluable connections in the field of geropsychology, and provided me with an amazing level of support and knowledge. I would also like to thank Paul Duberstein, Ph.D., and Silvia Sörensen, Ph.D., for assisting in my undergraduate experience. 
Finally, I would like to thank Evan Merz for his support throughout this project and throughout my experience in graduate school thus far. His ability to make me laugh and provide me with numerous reality checks has been, and will continue to be, invaluable. 


\section{Table of Contents}

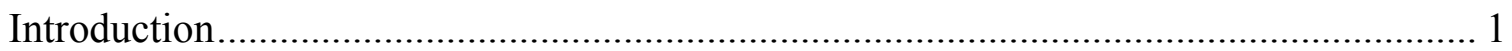

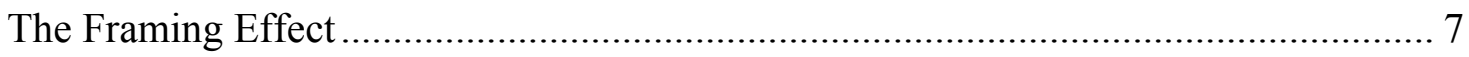

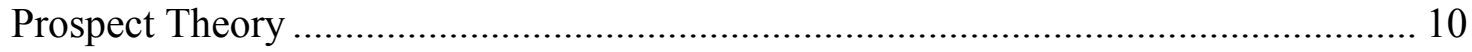

Medical Decision Making .................................................................................. 11

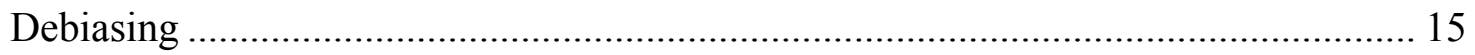

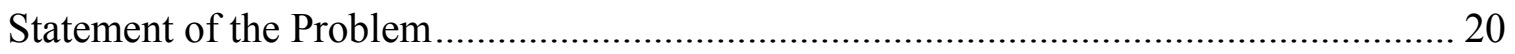

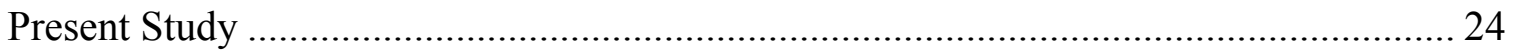

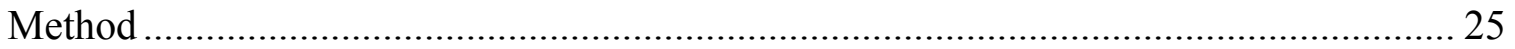

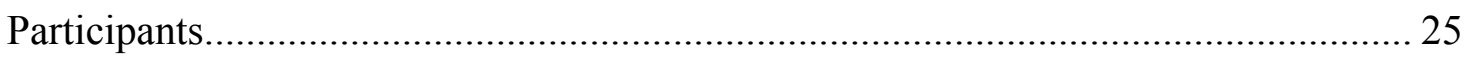

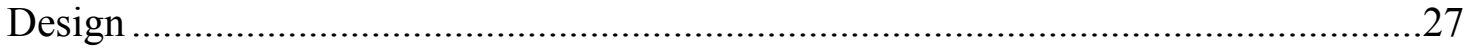

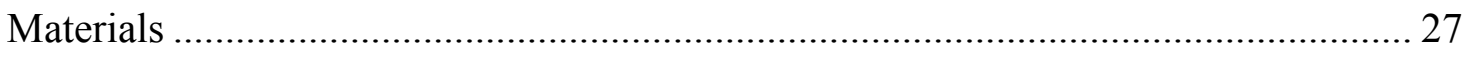

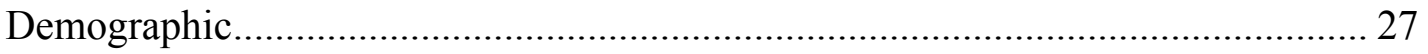

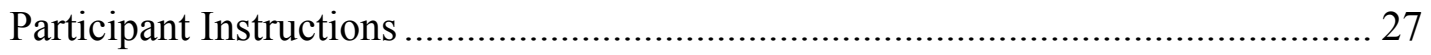

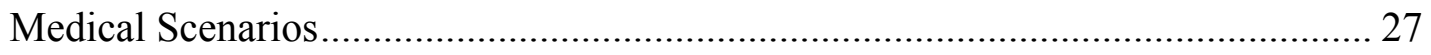

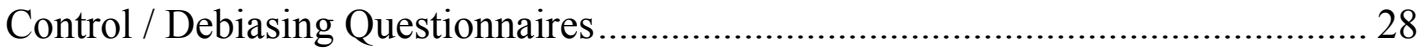

Treatment Choice Questionnaire .................................................................... 28

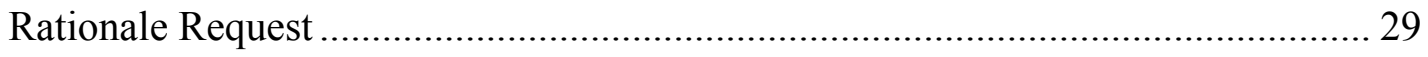

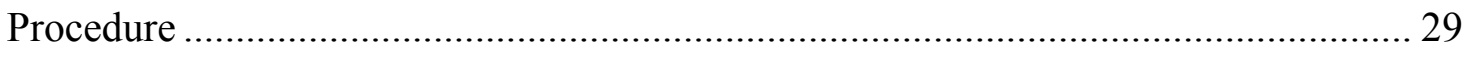

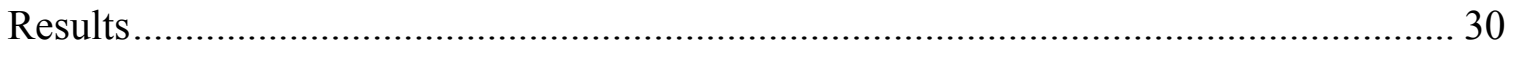

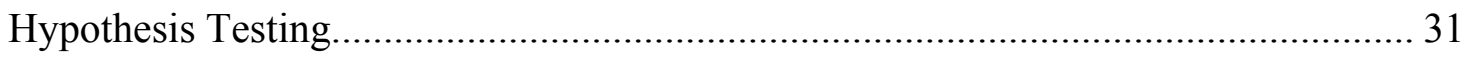

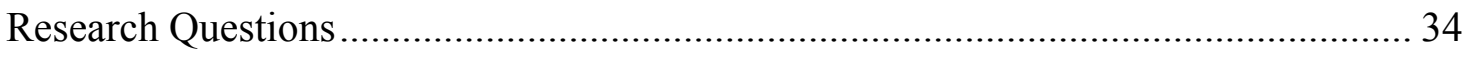

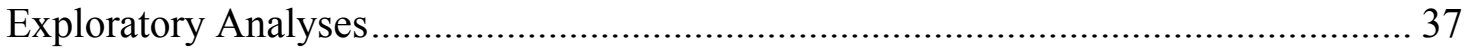




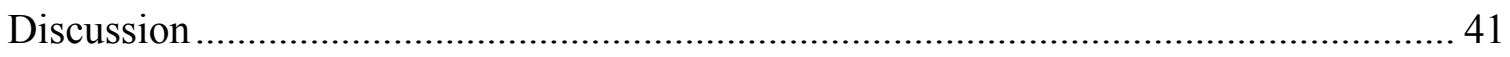

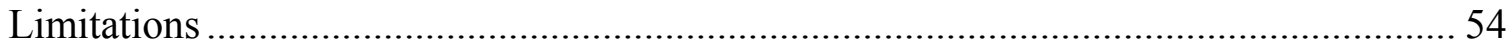

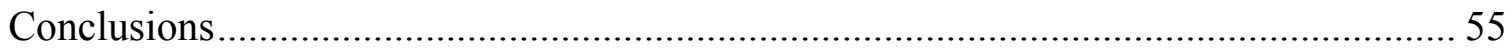

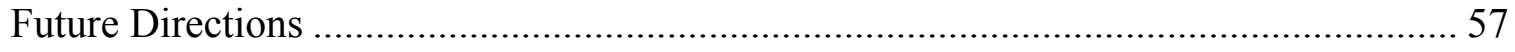

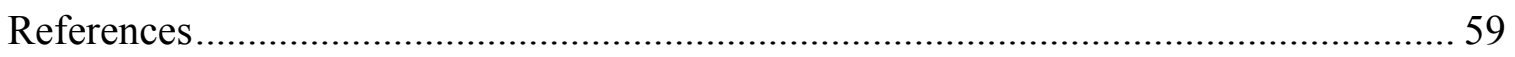

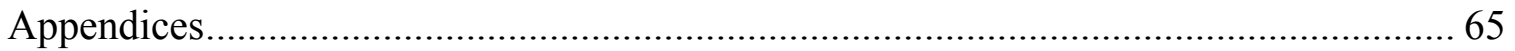




\section{List of Tables}

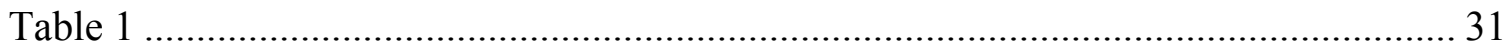

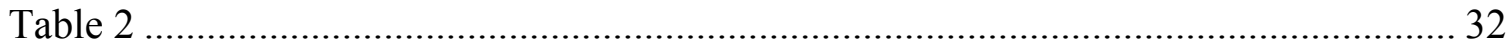

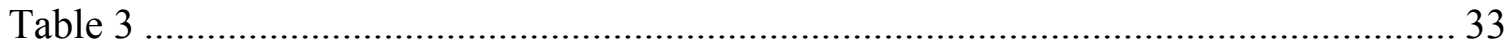

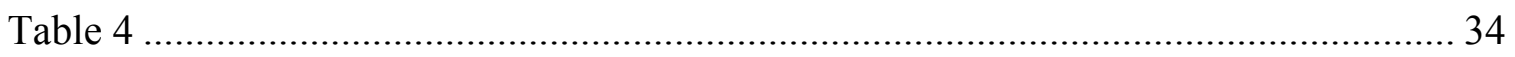

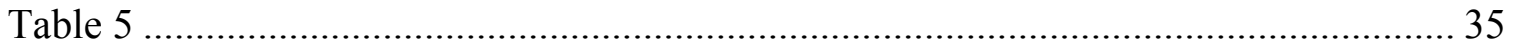

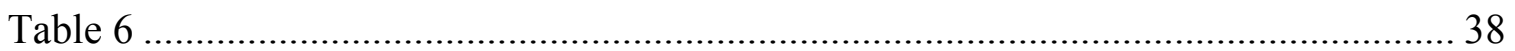

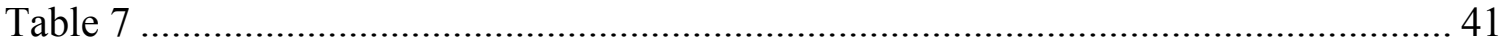




\section{Debiasing the Framing Effect in Younger and Older Adults'}

\section{Medical Decision Making}

Each day, we make countless decisions, ranging from decisions to perform everyday tasks, to decisions regarding major life changes. Although many of us would like to think that these decisions, whether small or large in scope, are based on rational consideration of the facts, many researchers (e.g., Tversky \& Kahneman, 1974; Fischhoff, 1975; Kahneman \& Tversky, 1984; Arkes \& Blumer, 1985; Miller \& Fagley, 1991) have demonstrated that this is frequently not the case. We tend to use heuristics, or mental shortcuts, which help us make relatively rapid decisions in situations where the information presented resembles that on which we have made previous decisions. The major advantages of using heuristics are the reduced processing load and increased speed. Without the use of heuristics, we would spend the majority of our day thinking about decisions that are normally made automatically or with little information processing (e.g., Schneider \& Shiffrin, 1977). A major disadvantage of using heuristics is that it can lead us to consider a limited amount of the available information, resulting in a less informed, and potentially suboptimal, decision.

In a classic article on decision making, Tversky and Kahneman (1974) described three of the most common heuristics individuals use when making a decision: the representativeness heuristic, the availability heuristic, and the adjustment and anchoring heuristic. Individuals often use the representativeness heuristic when they hear a description about a particular person (e.g., Ted likes to read, and would be described as quiet and reserved) and decide from this description that the person most likely fits into a particular category, such as "nerd." This decision is based on how representative the 
description of the person is of the stereotypes we may hold about nerds, rather than how probable it is that this person is actually a nerd. Tversky and Kahneman (1974) demonstrated the representativeness heuristic by giving participants brief personality descriptions of several individuals. The participants were then asked to assess the probability that each of these individuals held a certain occupation, based on a list of occupations presented to participants. A sample description was: "Steve is very shy and withdrawn, invariably helpful, but with little interest in people, or in the world of reality. A meek and tidy soul, he has a need for order and structure, and a passion for detail" (Tversky \& Kahneman, 1974, p.1124). Participants were most likely to choose librarian as the most probable occupation for Steve. This judgment was based on how representative Steve was of the stereotype the participants held about librarians (e.g., reserved and quiet), instead of basing the decision on facts that could inform the decision (e.g., the number of libraries in the area).

The availability heuristic occurs when a decision is made based on the ease with which an individual can retrieve information from long-term memory. For instance, one may make a decision about the prevalence of individuals with HIV/AIDS based on the number of individuals one has been exposed to with HIV/AIDS, without considering the actual prevalence of HIV/AIDS in the general population (i.e., base rate). This heuristic is demonstrated with an example from Hastie and Dawes (2001). When asked which is more common, murder or suicide, individuals often reply that murder is more common, even though statistics indicate that suicide is more common. The answer of murder is presumably given because murders often get more publicity than suicides, and therefore this information is more "available" than information on suicides. 
An example of the third heuristic, adjustment and anchoring, is provided by Chapman and Johnson (2001). Imagine you are trying to set a value for an antique piece of furniture you have just inherited. You have recently seen a piece like yours priced rather steeply at a local antiques dealer, so you price your piece at about the same price as theirs. However, if you had instead seen a similar piece at a yard sale for a much lower price, you would be more inclined to price your piece of furniture in accordance with that lower price. As implied by the name of this heuristic, an individual first recalls an "anchor," or a number he or she has in his or her head that may represent the appropriate number, and then adjusts that value up or down accordingly from this anchor. In the example above, being presented first with either a low or high anchor influences the final price chosen for the piece of furniture.

To test the adjustment and anchoring heuristic, Tversky and Kahneman (1974) asked participants to provide answers to various questions, and to state their answers in percentages. For example, participants were asked to estimate the percentage of African countries in the United Nations. Prior to giving their answer, participants were given a random number, generated by spinning a wheel, between 1 and 100 (the anchor). The participants were then asked to estimate the answer to the initial question. For those participants initially given the number 10 as their anchor, the median answer to the original question was 25 percent. For those participants initially given the number 65 as their anchor, the median answer to the original question was 45 percent. This example illustrates that the presentation of a high or low anchor is related to the answer that is provided. 
In addition to the influence of heuristics, our choices can be biased by how we use the relevant information we have stored in memory. Often the judgments we make are memory-based in the sense that the knowledge we often need is stored in long-term memory. We tend to recall information that is easiest to retrieve from long-term memory (Hastie \& Dawes, 2001) in the process of making decisions. Therefore, our decision making can be biased when it is based on the selective retrieval of information that is easiest to retrieve.

One of the common biases is the hindsight bias (Fischhoff, 1975). This bias occurs when individuals overestimate their ability to predict the correct outcome of situations in advance for which the outcome has been provided to them (Chapman \& Johnson, 2001). For example, a coworker may say that she knew "all along" that a certain candidate would win the election. However, if the same coworker was asked during the election which candidate she thought would win, she would not be as confident in the answer. The hindsight bias is a common attempt to have our memories of events fit our current knowledge of events (Hastie \& Dawes, 2001). The memory that is more accessible is who actually won the election. Trying to remember what our thought process was during the actual event requires more mental processing than assuming that what is correct now is what we thought all along.

Although recalling our easiest to remember memories may be an efficient use of time in the decision making process, it often leads to errors in judgment. In an attempt to organize the literature on judgment errors, Arkes (1991) developed a classification system for errors of judgment according to the "cost" each bias represents to a person. For example, the use of some heuristics has a benefit of faster processing speed, but a 
cost of reduced attention to details. Arkes' first category is strategy-based judgment errors. This type of judgment error involves trading the "cost" of complex cognitive processing for the benefit of reduced processing time, thereby making the complex task at hand less taxing to the individual. An example of a strategy-based judgment would involve increasing processing speed by overlooking necessary information in a complex problem solving task. Overlooking necessary information may lead to an incorrect answer due to the reduced time spent attending to the relevant details of the problem.

Arkes' second category is association-based judgment errors. This type of judgment error results from filtering information so it fits a pre-existing cognitive structure, thereby reducing processing time. The cost of this bias is that a certain hypothesis may be viewed as correct, simply because the information we need to evaluate the hypothesis is easy to retrieve from memory. Examples of association-based judgment errors are the representativeness and availability heuristics, and the hindsight bias, discussed above. All of these heuristics rely on pre-existing knowledge that individuals try to "fit" onto the current situation, even if it is incorrect for the current situation.

To demonstrate association-based judgment errors, Arkes (1991) provided an example of an experiment conducted by Gilovich (1981) in which newspaper sportswriters rated the potential of various college players to become professional football players. If a college player was said to come from the same hometown of a current professional football player, the college player was rated as having a much higher probability of becoming a professional football player than those college players who were not from the same hometown as a professional player. The sportswriters relied only on the information regarding which schools had previously produced professional 
football players, not on the actual data about the quality of the football program or the talent of the individual football player. The sportswriters were relying on the availability heuristic by assuming that certain schools would have higher rates of producing professional football players, only because the data on where the professional football players had attended high school was currently available to them.

Arkes' third category is psychophysically based errors. These errors result from the use of an unconscious reference point to which we orient when making a decision. Examples of psychophysically based errors include the anchoring and adjustment heuristic, the sunk cost effect, and the framing effect. The sunk cost effect, originally described by Arkes and Blumer (1985), results from decision making based on how much money has been spent on a project in the past. Arkes and Blumer provided an example of the sunk cost effect with the results of a field study about season ticket subscribers to a theatre series. In this study, individuals who paid more money for a season ticket subscription attended more plays during the next 6 months than those who had paid less money for a season ticket subscription, in order to justify the money they had already spent. The group who initially paid more felt like they had more of an investment in the tickets, rather than realizing that whether they attended the plays, the money had already been spent. The sunk cost effect occurred in this study due to the greater tendency of the higher-paying group to continue with the theatre series once they made a monetary investment in it, due to a desire to not appear wasteful, even though the money had already been spent regardless of whether they attended the theatre series. 


\section{The Framing Effect}

Another common error that occurs under conditions of uncertainty in decision making is the framing effect. The framing effect occurs when an individual is presented with two differently worded (framed) versions of a problem that describe identical outcomes, yet results in a different decision for each version of the problem (Hastie \& Dawes, 2001). The classic study that demonstrated the framing effect used the "Asian Disease Problem" (Tversky \& Kahneman, 1981). Two groups were presented with the same situation of the United States preparing for an outbreak of an unusual Asian disease that was expected to kill 600 people. The participants had to choose between two alternative programs to combat the disease. The first group of participants was presented with two programs that were worded in terms of gains, that is, lives saved. One of the program options (Program A) was worded as a "sure-bet," stating that if Program A was adopted, 200 people would be saved. The other program option (Program B) was worded as a more risky option, stating that if Program B was adopted, there was a 1 in 3 probability that 600 people would be saved and a 2 in 3 probability that no one would be saved. The participants were then asked to choose between these 2 program options. Participants were more likely to choose Program A, the "sure-bet," or risk-averse option, even though in both options the outcome was the same (200 saved in Program A, and one-third of 600 saved in Program B).

The second group of subjects was presented with two programs that were worded in terms of lives lost, presenting a "sure-bet" and a "risky" program option. The sure-bet option (Program C) stated that if Program C was adopted, 400 people would die. The risky option (Program D) stated that if Program D was adopted, there was a 1 in 3 
probability that nobody would die, and a 2 in 3 probability that 600 would die. For Program D, the 2 in 3 probability that 600 would die was equal to 400 dying if Program $\mathrm{C}$ was chosen. The subjects were then asked to choose which program option they would implement. The majority of participants in the second group, which was presented with the programs in a "lives lost," or mortality frame, chose the program that was risky (Program D), instead of the program that was a "sure-bet." The different program options presented to the two groups, although worded differently, had the same probability of occurring, yet the first group was risk-averse when presented with survival data, and the second group was risk-seeking when presented with mortality data.

The framing effect appears to occur equally with younger and older adults (Mayhorn, Fisk, \& Whittle, 2002; Rönnlund, Karlsson, Laggnäs, Larsson, \& Lindström, 2005). Rönnlund et al. (2005) examined the effects of framing on risky decision making in younger $(M=23.8)$ and older adults $(M=69.1)$. Both age groups were risk seeking after being presented with the negative frame, and risk averse when presented with the positive frame. This recent research supports the findings of Tversky and Kahneman's (1981) classic Asian disease scenario, and provides further evidence that the framing effect can be demonstrated across adult age groups.

In an effort to distinguish between different types of framing effects, Levin, Schneider, \& Gaeth (1998) proposed a typology of framing effects: risky choice framing, attribute framing, and goal framing. Risky choice framing was originally proposed by Tversky and Kahneman's (1981) “Asian Disease Problem,” which is discussed above. The findings are generally consistent for studies examining risky choice framing. 
Individuals are more likely to be risk seeking when the outcome is negatively framed (mortality rates) and risk averse when it is positively framed (survival rates).

The second type of framing outlined by Levin et al. (1998) is attribute framing. This occurs when the description of an object has either a negative or positive attribute associated with it. An example of this type of framing is evident in Levin's (1987) experiment in which subjects were asked to rate the desirability of ground beef based on whether the description of the beef emphasized the fat content or the lean meat content. Subjects were either told that the ground beef was $75 \%$ lean (positive frame) or $25 \%$ fat (negative frame). Subjects who were asked to rate the desirability of the $75 \%$ lean beef rated it as leaner, less greasy, and of higher quality than those subjects who were asked to rate the desirability of the $25 \%$ fat beef. Despite the fact that the total fat content was the same for the two descriptions, subjects presented with either of these two descriptions gave different ratings of the beef desirability. This study illustrates how even the framing of relatively objective information, such as fat content, has an effect on decision making.

Goal framing involves describing a goal, such as quitting smoking, desirable consequences of achieving the goal, and persuasive reasons why achieving the goal is beneficial. The emphasis of the presentation of information is either on the potential benefit of achieving the goal, or the potential of the goal to prevent a loss. This type of framing is illustrated nicely with a study by Meyerowitz and Chaiken (1987). The researchers presented participants with information regarding breast self-examination (BSE). Half of the participants received information that highlighted the negative consequences of not performing breast self-examination (negative frame). The other half of the participants received information on the benefits of performing breast self- 
examination (positive frame). The results indicated that, compared with the positively framed information, the negatively framed information (consequences of not doing the behavior) produced more favorable attitudes about performing BSE, greater estimates of number of times in the next year the participants actually intended to perform BSE, and a greater occurrence of actually performing BSE, as indicated in a 4-month follow-up interview.

\section{Prospect Theory}

In 1979, Kahneman and Tversky proposed prospect theory, which describes why individuals may succumb to the framing effect. Prospect theory stands out from other theories of economic utility because of the idea of a moveable reference point (Hastie \& Dawes, 2001). A moveable reference point means that for each decision making process, different standards are applied by the individual. What constitutes a loss in one situation could differ from what constitutes a loss in another situation. Another tenet of prospect theory is that losses hurt more than gains satisfy. Thus a gain of some monetary value is not equal to the loss of that same value. This is termed loss aversion. Finally, Kahneman and Tversky proposed that decision makers will be risk averse when deciding between gains, and risk seeking when deciding between losses. Hastie and Dawes (2001) provided an example of decision making with prospect theory:

Imagine you have just received $\$ 200$. Which of the following options would you prefer?

Option 1: You receive an additional $\$ 100$.

Option 2: A fair coin is tossed. If it lands heads, you receive an additional $\$ 200$; if it lands tails, you receive nothing more (p.216). 
When presented with the above scenario, most individuals choose Option 1, demonstrating that they are risk averse when it comes to deciding between gains. One would rather be sure he or she receives the additional money than take a risk with Option 2.

In an example representing risk seeking behavior, an individual is presented with this dilemma (Hastie \& Dawes, 2001):

Imagine you have just received $\$ 400$, but there is a penalty attached. You must choose one of the two penalty options:

Penalty Option 1: You must give back \$100.

Penalty Option 2: A fair coin is tossed. If it lands heads, you must give back $\$ 200$; if it lands tails, you may keep all of the money (p. 216).

When presented with this scenario, most respondents choose penalty option 2 , demonstrating that when presented with possible losses, most respondents are risk seeking.

The framing effect works in a similar way as the examples outlined above.

Subjects are presented with two scenarios with equal outcomes, though the outcomes are worded differently. Based on which wording the subject receives, different decisions are made. An area where the framing effect has been consistently demonstrated is that of medical decision making.

The Framing Effect and Medical Decision Making

Studies on framing in medical decision making indicate that patients may be influenced by the frame of the medical information presented for a decision, and may not be making entirely bias-free medical decisions (Hastie \& Dawes, 2001). In today's 
medical world, doctors are expected to present patients with a variety of options for treatment, along with information regarding prognosis and likelihood of desired and undesired results for each treatment. In the past, patients were typically not given a choice, and may not have even been informed why they were supposed to take a certain medication (Hastie \& Dawes, 2001). One may assume that being presented with treatment options would provide the basis for a bias-free decision, due to the doctor explaining the benefits and drawbacks of each treatment. However, several studies have shown that the way the medical information is presented can affect the final decision made by the patient.

How the physician describes the probability of different treatment outcomes can affect the patient's decision, even when different choices yield the same probability of a particular outcome (e.g., death or survival). For instance, wording the treatment outcome in terms of survival rates or in terms of mortality rates can affect which treatment the patient chooses (e.g., Moxey, O’Connell, McGettigan, \& Henry, 2003; Armstrong, Schwartz, Fitzgerald, Putt, \& Ubel, 2002; McKee, 2001; Tengs, 1987). In a classic demonstration of the effect of survival versus mortality wording, McNeil, Pauker, Sox, and Tversky (1982) presented physicians, patients, and graduate students with two medical procedures, radiation therapy and surgery, and asked them to choose between the two treatments for a hypothetical lung cancer patient. The two potential outcomes of the treatments (number living or dying within certain intervals of receiving the treatment) were presented either in survival or mortality wording. In both the survival and mortality worded scenarios, surgery was presented as having a more immediate risk than radiation. 
For example, in the survival wording, the surgery option stated that out of 100 people, 90 would live through the postoperative period, whereas the radiation option stated that out of 100 people, all would live through treatment. For the mortality wording, the survival option stated that out of 100 people, 10 would die during surgery or in the postoperative period, whereas the radiation option stated that out of 100 people, none would die during treatment. In the first scenario, worded in terms of survival rates, only $25 \%$ of respondents chose radiation. In the second scenario, worded in terms of mortality rates, $42 \%$ of the respondents chose radiation. This indicates that participants were more likely to choose surgery, the high-risk option with higher long-term survival rates, when information was presented in terms of survival, and choose radiation, the low-risk option with higher short-term survival rates, when information was presented in terms of mortality.

McNeil et al. (1982) also examined treatment preference under two different outcome information types: life expectancy and cumulative probability. They found that, across participants, surgery was chosen most often when outcome information was presented in terms of life expectancy.

Tengs (1987) performed a study similar to McNeil et al. (1982) in which undergraduate students were presented with six treatment scenarios. Three of the potential outcomes of the treatments were worded in terms of survival rates $(90 \%$ of people would live until the end of treatment), while the other three potential outcomes were worded in terms of mortality rates $(10 \%$ of people would die by the end of treatment). In both the mortality frame and survival frame, one scenario was worded in terms of cumulative probability (e.g., 10 will die in treatment, 32 will have died by 1 
year), one scenario was worded in terms of interval probability (e.g., 22 people will die in the interval between treatment and one year), and one scenario was worded in terms of overall life expectancy (e.g., the number of people who live 1 to 5 years after treatment). Tengs found that subjects more often chose surgery (the high-risk option) in the survival frame and radiation (the low-risk option) in the mortality frame, when data were presented in the cumulative probability format. The subjects chose surgery least often when data were presented in the life expectancy format. Therefore, both the outcome information type (cumulative probability, interval probability, or life-expectancy) and the frame (survival or mortality) had an impact on the medical treatment chosen.

McKee (2001) replicated and extended the work of Tengs (1987) to demonstrate the framing effect in medical decision making among both younger and older adults. Young college students and older adults were presented with the same six medical scenarios as used in Tengs' (1987) study. However, in McKee's (2001) study, each subject was presented with both the survival and mortality wording, in a within-subjects design. McKee (2001) also investigated the effects of a physician recommendation on the framing effect, since physicians are often looked to as experts and are expected to recommend what they consider to be the best course of action for their patients. The results of the study found that across age groups and outcome information types, surgery (the high-risk option) was chosen more frequently than radiation (the low-risk option) in the survival frame, and radiation was chosen more frequently than surgery in the mortality frame. This finding is consistent with the results of McNeil, Pauker, Sox, and Tversky (1982). Additionally, McKee (2001) found a framing effect for survival versus mortality wording when outcome data were presented in the life expectancy format. In 
these data formats, both older and younger adults chose surgery significantly more often than radiation. The physician recommendation did not eliminate the framing effect.

The results of these studies indicate that patients may not be making bias-free decisions when presented with a few options for treatment, given that respondents make different decisions when the information is presented in various frames and outcome information types. Patients may potentially choose a more risky option when the information is presented in terms of survival rates. Additionally, how the outcome information is presented (cumulative probability, interval probability, or life-expectancy) has an effect on the final decision made by a patient.

In light of the many studies showing support for a framing effect in medical decision making, one may wonder whether it is possible for a patient to make an informed decision regarding which medical treatment is objectively the best one for him or her. All of the studies discussed above reveal that patients make different treatment decisions depending on how the treatment is framed. This could lead a patient to choose a treatment based only on wording, with no information on which treatment is objectively better. One option that gives control to the patient is to inform patients of the framing effect and attempt to teach them a strategy that avoids the framing effect, that is, a debiasing strategy.

\section{Debiasing}

A small amount of literature has been devoted to the task of debiasing individuals against the various biases that lead to errors in judgment. However, as Turk and Salovey (1986) noted, most of the literature on judgment biases has identified various forms of bias (e.g., Thorburn, Harvey, \& Ryan, 2005; Reimer, Mata, \& Stoecklin, 2004), 
but has not addressed how to avoid the bias. One might think that simply by describing a bias to an individual, the individual will become aware of the bias, and therefore not exhibit the bias when making a judgment. However, as Arkes (1981) pointed out, this approach has not proven effective. Fischhoff (1977) demonstrated the ineffectiveness of this approach by presenting two groups of subjects with a series of difficult two-choice questions, where the correct answer was already circled for them. The subjects were asked to answer the questions as if they had been given them without the correct answer circled. Having the correct answers already circled may lead the subjects to commit the hindsight bias, which would lead them to overestimate their ability to predict the correct answer when it has already been provided for them. One of the groups was provided with a description of the hindsight bias and how this bias could affect their response to the questions. The second group was not informed of their potential to commit the hindsight bias. The first group was asked to try and not commit the same error of judgment that participants in the second group would commit by knowing about the hindsight bias. The attempt at debiasing was unsuccessful, as both groups exhibited the hindsight bias.

One debiasing technique that has been shown to work across a wide range of scenarios involves asking participants to provide a rationale for their answers. In a demonstration of this debiasing technique, Arkes, Faust, Guilmette, and Hart (1988) were able to successfully eliminate the hindsight bias by asking participants to state one reason supporting the final choice they made. In this study, three groups of neuropsychologists were presented with three possible diagnoses for a short case history. One group of subjects was told which of the three diagnoses was the correct one, while a second group 
was not told which diagnosis was the correct one. The participants were then asked to assign a probability to each of the three choices that they would have given each of the diagnoses based on the symptoms, before being told the correct diagnosis. The group that was told of the correct diagnosis showed a hindsight bias towards that diagnosis, assigning a larger percentage to the correct diagnosis. A third group of subjects, who were also given the correct diagnosis from the start of the study, were successfully debiased against the hindsight bias by stating one reason supporting each of their three diagnoses, before assigning probabilities.

The framing effect has received less attention than other judgment biases with regard to debiasing. Only a few studies to date (Miller \& Fagley, 1991; Sieck \& Yates, 1997; Bernstein, Chapman, \& Elstein, 1999; Mashat, 2004) have attempted to neutralize the framing effect through debiasing. One of these studies (Miller \& Fagley, 1991) successfully debiased individuals against the framing effect by requesting a rationale for their choices, as was done by Arkes et al. (1988) with the hindsight bias. The participants were presented with six scenarios similar to the scenarios used in Tversky and Kahneman's (1981) Asian disease scenario. Half of the participants were asked to make a choice between intervention programs after reading the scenarios. The other half were asked to provide a rationale before making their final choice. In spite of the equivalence of the two intervention programs, the participants who did not provide a rationale chose the more risky program when the scenarios were framed negatively rather than positively, thus demonstrating the framing effect. The participants who were asked to provide a rationale chose program options with similar frequency when presented with the positive and negative frames. By having to justify the decision they were making, the debiased 
group of subjects became aware of the equivalence of the two programs and the framing effect was eliminated.

Sieck and Yates (1997) used a similar procedure to preclude the framing effect in college-aged students presented with the Asian disease scenario and a scholarship problem scenario. Participants were placed in either a control, anticipated exposition, or exposition group. Participants in the exposition group were asked to write why they thought their choice was the "smart thing to do" before making their choices. Participants in the anticipated exposition group were told that they would be asked to write about their choice after they had made it. The control group participants were not instructed to write about their decisions. For the Asian disease scenario, the framing effect was not present in the exposition group, but was present in the anticipated exposition and control group. For the scholarship problem scenario, which was chosen because it is not as strict a representation of the framing effect as is the Asian disease scenario, none of the conditions successfully eliminated the framing effect. Although the results of this study are confusing in light of the different results for the Asian disease and scholarship problem scenarios, the mechanism of debiasing is similar to Miller and Fagley's (1991) rationale request technique.

Bernstein, Chapman, and Elstein (1999) attempted to debias participants against the effect of framing in a clinical context. Participants were asked to imagine that they had a chronic disease that would result in them living for about two years if the disease went untreated. They were then told of a drug that allowed patients to live for about 20 years with the disease. However, for this drug to work, it had to be paired with one of two supplementary drugs that induced life-shortening diseases. Participants were then 
asked to choose one of the supplementary drugs. One group of participants received segregated editing, in which the outcomes of the supplementary drug were presented alone, forcing the participants to determine the additive effects of the original and supplementary drugs. A second group received integrated wording, in which the original drug effects were combined with the supplementary drug effects to produce a final estimate of longevity. A framing effect was found between these equal, but differently worded, scenarios. A third group of participants received both the segregated editing and the integrated editing formats, in a within-subjects design. Results showed that presenting both alternative editings prior to asking the participant for his or her choice helped to reduce the effect of frame.

In a more recent study, Mashat (2004) attempted to debias college-aged students against the framing effect. Using the same six medical decision scenarios used by Tengs (1987) and McKee (2001), participants were either presented with survival or mortality worded scenarios, with one scenario presented in cumulative probability format, one in interval probability format, and one in overall life-expectancy format. Before making their choices, half of the participants received a debiasing questionnaire asking them to consider the advantages and disadvantages of surgery vs. radiation. The other participants (control group) received a questionnaire containing general information related to cancer. This questionnaire was provided as a control for time and effort, and the participants were not asked to do anything with this information.

When the data were presented in cumulative probability format, participants more often chose surgery in the survival worded scenario and radiation in the mortality worded scenario. Therefore, a framing effect was found for the cumulative probability format, as 
indicated by the discrepancy in treatment choice based on survival or mortality wording. A framing effect was not found for the scenarios that were worded in interval probability format or overall life expectancy format. Because a framing effect was shown only for the scenario worded in terms of cumulative probability, this scenario was the only one tested for successful debiasing. For the cumulative probability data format, those participants who received the debiasing questionnaire did not show a framing effect for survival versus mortality wording. That is, there was no significant difference in the proportion of participants who chose surgery or radiation in the survival worded or mortality worded scenarios.

The results of these debiasing studies indicate that the use of heuristics and automatic processing, which lightens the cognitive load and shortens the mental processing time, often leads to errors in judgment, which can be circumvented by directly asking individuals to provide reasons for their choices. By using the "rationale requested" technique, the framing effect was eliminated in the debiasing studies. This allowed the participants to make medical decisions without the biases associated with the framing of medical information.

\section{Statement of the Problem}

The population of older adults is steadily increasing. In 2002, those over the age of 65 comprised over $12 \%$ of the U.S. population. This number is expected to increase as the "Baby Boom" cohort begins to age. By the year 2030 the percentage of the U.S. population over the age of 65 is projected to jump 20\% (U.S. Administration on Aging, 2003). Although approximately $38 \%$ of non-institutionalized older adults report their health as being "excellent" or "very good" (U.S. Administration on Aging, 2003), 85\% of 
older adults over the age of 60 have at least one chronic disease (Cassel, Rudberg, \& Olshansky, 1992). As one ages, the increased risk of disease leads to the increased demand to make important medical decisions. Older age also increases the likelihood that an individual will face long term care, where decisions are often made regarding medical advance directives. Therefore, older adults are potentially making more medical decisions than any other age group. Additionally, older adults are more likely to face medical decisions that have potentially life-threatening consequences.

Taking into consideration the results of the debiasing studies presented, it may be possible to eliminate decisional biases that are operative (e.g., framing effect, representativeness heuristic). Because the framing effect results from the method by which information is presented (e.g., Kahneman \& Tversky, 1984; McKee, 2001; Rönnlund et al., 2005), there is an opportunity for an intervention that could yield better informed and less biased medical decisions.

As discussed earlier, Mashat (2004) used a questionnaire that required young adult participants to consider the potential advantages and disadvantages of a hypothetical medical treatment, to preclude the effect of framing. Similarly, Miller and Fagley (1991) requested a rationale from participants before making a choice, whereas Sieck and Yates (1997) asked the participant to write about their choice before making it. These techniques that required careful consideration of alternatives successfully eliminated the framing effect. Bernstein, Chapman, and Elstein (1999) presented each participant with two frames, instead of one, to reduce the framing effect. They hypothesized that seeing two different frames of the decision would allow the participant to compare the discrepant information and therefore eliminate the framing effect. 
Although Bernstein, Chapman, and Elstein (1999) reported that the framing effect was reduced when participants received two frames, McKee (2001) found that the framing effect was produced with certain outcome information types when participants were presented with two frames. Additionally, Mashat (2004) was only able to produce a framing effect when data were presented in the cumulative probability format, while McKee (2001) found an effect of frame for both younger and older adults when the data were presented in life expectancy format.

In light of the many healthcare decisions faced by older adults, and the potential gravity of their consequences, finding successful ways to avoid the framing effect in medical decision making is especially important for this population. Though Mashat (2004), Bernstein, Chapman, and, Elstein (1999), and Miller \& Fagley (1991) demonstrated debiasing with young adults, no published research has attempted to preclude the framing effect through a debiasing procedure with older adults. All of the debiasing studies to date have been performed with college-aged younger adults. Because of this, nothing is known about whether other age groups would respond similarly to a debiasing procedure. There are a variety of ways that younger adults may differ from older adults in medical decision making. Younger adults tend to be a relatively healthy population, so medical decision making scenarios, often involving lifethreatening disorders, may not be as salient to them as they would be to older individuals who are more likely to face medical decisions. Because of the relative healthiness of younger adults, they may not have as much experience with making medical decisions as an older population. In addition, older adults may be more equipped to evaluate different pieces of information upon which a medical decision is to be made. For example, older 
adults have probably accumulated more information about medical disorders due to the accumulated number of medial problems over their lifespan and the fact that most have at least one chronic illness. Additionally, older adults potentially have more exposure to, and discussions with, friends and relatives that have illnesses.

Yates and Patalano (1999) present a model of analytic, rule-based, and automatic processing which states that individuals presented with a decision for the first time are more likely to approach it analytically, whereas individuals who have seen situations repeatedly may invoke rule-based or automatic approaches to decision making. In medical decision making, older adults may be the more experienced decision makers, and therefore may approach decision making from a rule-based, or automatic approach, which would make them more likely to use heuristics. It is unclear, however, whether these age-related differences influence susceptibility to the framing effect or the outcome of attempts to preclude the effect.

Younger and older adults may also differ with regard to some of the cognitive abilities used for decision making (e.g., working memory, reasoning; Craik, 2000), which could contribute to age-related differences in the decision making process. For example, medical decision making often demands considerable working memory and processing resources. Compared to younger adults, older adults often show poorer performance on working memory tasks (Park et al., 1996), comprehension tasks (Dixon, Hultsch, Simon, \& von Eye, 1984), and processing speed tasks (Salthouse, 1993). In light of these declines in cognitive functioning, some have argued that older adults may rely more on heuristics during decision making to reduce the cognitive demands of the task (e.g., Park, 1999). Consequently, older adults may be more susceptible to the framing effect because 
they are less inclined to consider and process all medical information presented to them. On the other hand, one might expect older adults to be less susceptible to the framing effect because they are more likely to have the relevant knowledge necessary to make unbiased medical decisions.

Currently, none of the research on the framing effect has attempted to determine who is more likely to succumb to the effect of framing or to the debiasing procedure. In Mashat's (2004) study, not all participants succumbed to the effect of framing, and not all participants were able to be debiased. One component of the decision making process that may relate to susceptibility to framing and debiasing is how complete an individual's rationale is for choosing one treatment over another. Meyer, Russo, and Talbot (1995) found that older women offered less complete rationales for their decisions regarding breast cancer treatment options, indicating that a participants' rationale may play a part in the decision making process.

\section{Present Study}

The present study replicated and extended Mashat (2004) and McKee (2001) by testing the following hypotheses: 1a) the proportion of younger adults in the control group selecting surgery in the survival frame will differ significantly from the proportion selecting surgery in the mortality frame in at least one outcome information type, indicating an effect of frame, $1 b$ ) the proportion of older adults in the control group selecting surgery in the survival frame will differ significantly from the proportion selecting surgery in the mortality frame in at least one outcome information type, indicating an effect of frame, 2a) the proportion of younger adult participants in the debiasing group selecting surgery in the survival frame will not differ from the proportion 
selecting surgery in the mortality frame, indicating a successful debiasing procedure, and 2b) the proportion of older adult participants in the debiasing group selecting surgery in the survival frame will not differ from the proportion selecting surgery in the mortality frame, indicating a successful debiasing procedure.

A number of research questions were examined since the previous literature examining these topics was mixed. The first research question asked: is the proportion of older adults who succumbed to the effect of framing, as indicated by a choice reversal across 2 or more scenarios, different than the proportion of younger adults who succumbed to the effect of frame? The second question asked whether sex and age interacted as predictors of the framing effect. The third question asked whether the relevance of the reasons provided on the debiasing questionnaire predicted a framing effect.

\section{Participants}

Method

Two groups, younger and older adults, were recruited for participation in the current study. The first group consisted of 63 younger adults (38 women and 23 men) recruited from Introduction to Psychology classes, who had a mean age of 18.6 years (range 18-30; $S D=1.9$ ). Their mean education level was 13.3 years (range $11-17 ; S D=$ 1.0). The majority of the younger adult participants (55.6\%) had been examined by a physician in the last 2 to 6 months. A large percentage of the younger adults were Caucasian (79.4\%), single (95.2\%), and reported their health status as "Good" $(52.4 \%)$, with only $3.2 \%$ reporting that they had been diagnosed with a chronic illness. A moderate percentage of younger adults reported that they currently smoked $(15.9 \%)$. In terms of experience with cancer, $74.6 \%$ of the younger adults reported that they knew 
someone who had been diagnosed with cancer. The majority of this group knew someone who had been diagnosed with a cancer other than lung cancer (52.4\%). For $63.5 \%$ of those who knew someone with cancer, the person was in their extended family. Approximately half of the younger adult participants had discussed the treatment of cancer with someone who went through it (49.2\%).

The second group of participants consisted of 61 older adults (41 women and 17 men), recruited from senior centers and assisted living facilities, who had a mean age of 76.8 years (range $65-98, S D=7.1$ ). Their average level of education was 12.7 years (range $8-21, S D=2.9)$. The majority of older adult participants $(52.5 \%)$ had been examined by a physician within 2 months of their participation in the study. Most of the older adult participants were Caucasian (95.1\%), widowed (57.4\%), and reported their health status as either "Good" (39.3\%) or "Average" (29.5\%), with 65.6\% reporting that they had been diagnosed with a chronic illness, and $4.9 \%$ reporting that they currently smoked. A large percentage of the older adults $(82.0 \%)$ reported that they knew someone who had been diagnosed with cancer. For the majority of these participants $(65.6 \%)$ the individual with cancer was in their immediate family, with $19.7 \%$ reporting that this individual had been diagnosed with lung cancer. Among the older adult participants, $60.7 \%$ had received or provided care at some point in their lives. The majority of these participants were the care provider (54.1\%), and had been the provider for anywhere between less than 6 months (18.0\%) for more than ten years (3.3\%). Many of the older adults $(59.0 \%)$ reported never discussing the treatment of cancer with an individual going through treatment. 


\section{Design}

A 2 (age) x 2 (frame) x 3 (data format) design was employed, with repeated measures over the data format variable. All participants received three medical scenarios: one in the cumulative probability data format, one in the interval probability data format, and one in the life expectancy data format. Half of the participants received the three medical scenarios in survival wording, and half received the three medical scenarios in mortality wording.

\section{Materials}

Demographic. The demographic questionnaire (Appendix A) used in the current study was similar to the demographic questionnaire used by McKee (2001) and Mashat (2004). The questionnaire asked the participant to complete basic information such as age, sex, years of education, marital status, and ethnicity. The demographic questionnaire also included questions on the vicarious experience of cancer.

Participant Instructions. The participant instructions (Appendix B) provided the participant with background information on the two treatment choices, surgery and radiation. It also provided information on how well patients had recovered 6 weeks after receiving either treatment. It then asked the participant to answer the questions in the order they appeared and to not revisit questions once they had provided an answer.

Medical Scenarios. A set of three medical scenarios (Appendices C \& D) containing information about treatments for lung cancer, taken from Tengs (1987), McKee (2001), and Mashat (2004) served as the stimulus materials. The three scenarios were framed in either survival or mortality wording. The scenarios presented information on two alternative treatments for lung cancer: radiation treatment or surgery. The 
information about the treatments in the three scenarios was presented in cumulative probability, interval probability, and overall life-expectancy format. Therefore, there were 6 possible scenarios: survival wording, cumulative probability format; survival wording, interval probability format; survival wording, life-expectancy format; mortality wording, cumulative probability format; mortality wording, interval probability format; and, mortality wording, life-expectancy format.

Control or Debiasing Questionnaires. Participants were presented with either a debiasing (Appendix E) or control questionnaire (Appendix F). The debiasing questionnaire included 5 questions, which required that the participant list the advantages and disadvantages of each treatment, and the information that was the most relevant in making his or her treatment choice. The questions remained the same after the presentation of each scenario. This debiasing technique of requesting a rationale was similar to the technique used by Miller \& Fagley (1991) and Mashat (2004), to preclude the framing effect. There were 3 different versions of the control questionnaire. These questionnaires provided short commentaries on stress, dental hygiene, and physical fitness, and then asked the participant what changes he or she would be willing to make in his or her life if he or she was suffering from problems related to stress, gum disease, or lack of physical fitness. These questionnaires were chosen to provide a control for the time and cognitive effort that the debiasing group of participants invested in their questionnaire. These questionnaires contained no information related to any of the medical scenarios that could potentially affect the control group's treatment choice.

Treatment Choice Questionnaire. This form (Appendix G) asked the participants to circle whether they chose surgery or radiation for the scenario they just read. 
Rationale Request. After completing the treatment questionnaires, the control group participants were asked to record their treatment choice for all 3 scenarios on an answer sheet (Appendix H). These participants were then asked to fill in a form requesting that they detail which pieces of information were most important in making their treatment decision in the three scenarios (Appendix I).

\section{Procedure}

As participants volunteered to participate, they were sequentially assigned to one of four groups: survival worded scenarios with the control questionnaire, survival worded scenarios with the debiasing questionnaire, mortality worded scenarios with the control questionnaire, or mortality worded scenarios with the debiasing questionnaire. All participants were presented with 3 scenarios: 1 scenario for each outcome information type (cumulative probability, interval probability, and life-expectancy). After completing the written consent form and completing the demographic questionnaire, the participants read the participant instructions, and then read the first scenario. After reading the first scenario, the participants completed either the debiasing or control questionnaire, and circled their treatment choice (surgery or radiation; Appendix H). This process was repeated for the remaining 2 scenarios. The cumulative probabilities format was presented first, followed by the interval probabilities format and the overall life expectancy format.

Those participants who received the debiasing questionnaire were presented with the same questions after each vignette, while those who were presented with the control questionnaire received the stress questionnaire first, followed by the gum disease and physical fitness questionnaires. The debiasing group was finished with the study after 
completing the decision scenarios and making their treatment choices. Members of the control group were asked to raise their hands when they had finished completing their medical decision scenarios. A research assistant then removed the scenarios and used the answer sheet to circle the treatment choices the participant made. The participants then received the answer sheet with their treatment choices circled. At this point, the control group participants were asked to provide a rationale for the treatment choice they made on each of the 3 scenarios.

The participants were presented with the following materials in the following order: (a) cover letter (Appendix J), (b) written consent form (Appendix K), (c) demographic questionnaire, (d) participant instructions containing information about radiation and surgery, (e) three scenarios, framed in either survival or mortality data, (f) debiasing or control questionnaire, and (e) answer sheet and rationale request form for those participants in the control group. Data was collected in a group format, with approximately 5-10 participants per group administration. Upon completion of the study, younger adult participants were awarded extra credit for their participation. Older adult participants entered their names into a drawing for an opportunity to win $\$ 100$.

Results

\section{Initial Analyses}

Before beginning analyses, the control and debias groups were compared on demographic variables, to ensure that the groups were not significantly different on relevant variables. The average years of education for the control group $(M=13.04)$ did not differ significantly from that of the debias group $(M=13.01 ; t(112)=.07, p=.95)$. Additionally, the self-rated health status did not differ between the control $(M=3.75)$ and 
the debias groups $(M=3.84 ; t(120)=.50, p=.62)$. The control group was $64.4 \%$ female, whereas the debias group was $68.3 \%$ female. Within each group approximately equal percentages of participants reported being diagnosed with a chronic illness $(35.5 \%$ control, $32.3 \%$ debias), whereas slightly more control group participants reported providing care at some point in their lives (43.5\% control, $35.5 \%$ debias).

\section{Hypothesis Testing}

Hypothesis 1a was that the younger adult control group would show a framing effect in at least one of the three medical scenarios. This hypothesis was supported. The percentages for each treatment choice by format for the younger adult control group are presented in Table 1.

\section{Table 1}

Choice Percentages and Significance for Younger Adult Control Group

\begin{tabular}{lcccccc}
\cline { 2 - 6 } & $\begin{array}{c}\text { Cumulative Probability* } \\
(n=32)\end{array}$ & $\begin{array}{c}\text { Interval Probability* } \\
(n=32)\end{array}$ & $\begin{array}{c}\text { Life Expectancy } \\
(n=32)\end{array}$ \\
\hline Frame & Surgery & Radiation & Surgery & Radiation & Surgery & Radiation \\
\hline Survival & 68.8 & 31.3 & 81.3 & 18.8 & 62.5 & 37.5 \\
Mortality & 31.3 & 68.8 & 43.8 & 56.3 & 56.3 & 43.8 \\
& & & & & &
\end{tabular}

$* p<.05$; Fisher's exact test

In order to show the framing effect, significantly more participants than expected would have to choose surgery in the survival frame and radiation in the mortality frame. Therefore, a significant one-sided Fisher's exact test (FET) would indicate an effect of frame. For the cumulative probability format, the younger adult control group showed the framing effect $(p<.05 ; \mathrm{FET})$. A measure of the strength of association indicated that 
approximately $14 \%$ of the variance in treatment choice for this format could be explained by frame $(\mathrm{phi}=.375, p<.05)$.

For the interval probability format, the younger adult control group also showed a framing effect $(p<.05$; FET) with approximately $15 \%$ of the variance in treatment choice explained by frame $(\mathrm{phi}=.387, p<.05)$. Finally, for the overall life expectancy format, the younger adult control group did not show a framing effect ( $p=.500 ;$ FET).

Hypothesis $1 \mathrm{~b}$ was that the older adult control group would show a framing effect in at least one of the data formats. As stated above, a significant Fisher's exact test would indicate an effect of frame, due to the observed counts in the specified cells differing significantly from the expected counts. The second hypothesis was also supported. Treatment choice percentages by data format for the older adult control group are presented in Table 2.

Table 2

Choice Percentages and Significance for Older Adult Control Group

\begin{tabular}{|c|c|c|c|c|c|c|}
\hline \multirow[b]{2}{*}{ Frame } & \multicolumn{2}{|c|}{$\begin{array}{l}\text { Cumulative Probability } \\
\qquad(n=28)\end{array}$} & \multicolumn{2}{|c|}{$\begin{array}{c}\text { Interval Probability* } \\
\qquad(n=28)\end{array}$} & \multicolumn{2}{|c|}{$\begin{array}{l}\text { Life Expectancy } \\
\quad(n=29)\end{array}$} \\
\hline & Surgery & Radiation & Surgery & Radiation & Surgery & Radiation \\
\hline Survival & 53.3 & 46.7 & 71.4 & 28.6 & 73.3 & 26.7 \\
\hline Mortality & 53.8 & 46.2 & 28.6 & 71.4 & 50.0 & 50.0 \\
\hline
\end{tabular}

$* p<.05 ;$ Fisher's exact test

Whereas the younger adult control group showed the framing effect in 2 of the 3 scenarios (cumulative probability and interval probability), the older adult control group showed the framing effect in only one scenario (interval probability, $p<.05$; FET). For this data format, approximately $18 \%$ of the variance in treatment choice was explained by 
frame (phi $=.429, p<.05 ; \mathrm{FET})$. The cumulative probability format and the overall life expectancy format did not show a framing effect for the older adult control group $(p=$ $.638, .181$, respectively; FET).

Hypothesis $2 \mathrm{a}$ was that the younger adult group would avoid the framing effect through the use of a debiasing questionnaire. To show a successful avoidance of the framing effect, the proportion of participants choosing surgery in the survival frame and the proportion choosing radiation in the mortality frame would not differ significantly from the expected counts. Therefore, a non-significant one-sided Fisher's exact test would indicate a successful debiasing attempt. Treatment choice percentages are presented in Table 3.

Table 3

Choice Percentages and Significance for Younger Adult Debias Group

\begin{tabular}{lcccccc}
\cline { 2 - 6 } & $\begin{array}{c}\text { Cumulative Probability } \\
(n=31)\end{array}$ & $\begin{array}{c}\text { Interval Probability } \\
(n=31)\end{array}$ & $\begin{array}{c}\text { Life Expectancy } \\
(n=31)\end{array}$ \\
\hline Frame & Surgery & Radiation & Surgery & Radiation & Surgery & Radiation \\
\hline Survival & 53.3 & 46.7 & 66.7 & 33.3 & 80.0 & 20.0 \\
Mortality & 31.3 & 68.8 & 50.0 & 50.0 & 62.5 & 37.5 \\
\hline
\end{tabular}

For the cumulative probability format, the younger adult control group did show a framing effect and this effect was not present in the debiasing group $(p=.189$; FET). For the interval probability format, the younger adult control group also showed a framing effect and this effect was not shown in the debiasing group $(p=.283$; FET). For the life expectancy format, the younger adult control group did not show a framing effect, and there was also no framing effect demonstrated in the debiasing group ( $p=.250$; FET). 
Hypothesis $2 b$ was that the older adult group would avoid the framing effect through the use of a debiasing questionnaire. Treatment choice percentages by data format are presented in Table 4.

Table 4

Contingency Tables and Significance for Older Adult Debias Group

\begin{tabular}{lcccccc} 
& \multicolumn{2}{c}{$\begin{array}{c}\text { Cumulative Probability* } \\
(n=29)\end{array}$} & $\begin{array}{c}\text { Interval Probability* } \\
(n=27)\end{array}$ & \multicolumn{2}{c}{$\begin{array}{c}\text { Life Expectancy } \\
(n=27)\end{array}$} \\
\hline Frame & Surgery & Radiation & Surgery & Radiation & Surgery & Radiation \\
\hline Survival & 71.4 & 28.6 & 78.6 & 21.4 & 78.6 & 21.4 \\
Mortality & 26.7 & 73.3 & 38.5 & 61.5 & 61.5 & 38.5 \\
\hline
\end{tabular}

$* p<.05$; Fisher's exact test

For the cumulative probability format, the older adult control group did not show a framing effect, but a framing effect was present in the debiasing group for this format $(p<.05 ; \mathrm{FET})$. For the interval probability format, the older adult control group did show a framing effect, and it was not eliminated in the debiasing group $(p<.05$; FET). For the life expectancy format, the older adult control group did not show a framing effect, and the framing effect was also not present in the debiasing group $(p=.293)$. The raw data for the hypotheses $1 \mathrm{a}$ to $2 \mathrm{~b}$ are presented in Appendix $\mathrm{L}$.

Research Questions

Three research questions were examined since the literature in these areas was not conclusive enough to propose a directional hypothesis. The first research question asked which age group was most likely to succumb to the framing effect. The results are presented in Table 5. 
Table 5

Percentages for Succumbing to the Framing Effect by Age Group

\begin{tabular}{lcc}
\hline Age Group $(\mathrm{N}=117)$ & Did Succumb & Did Not Succumb \\
\hline Younger Adults & 45.2 & 54.8 \\
Older Adults & 29.1 & 70.9
\end{tabular}

Note. $\chi 2(1, N=117)=3.21, p=.07$

To test this research question, participants were coded as either showing the framing effect or not by determining whether the participant had a choice reversal at any point throughout the 3 scenarios. If all of a participant's treatment choices were the same across scenarios, he or she was coded as not showing the framing effect. If there was a choice reversal, he or she was coded as showing the framing effect. Therefore, a $2 \times 2$ chisquare was used to test this question (age x succumbing to the framing effect). The chisquare test was non-significant, $\chi 2(1, N=117)=3.21, p=.07$. An examination of the contingency table revealed that approximately $45 \%$ of younger adults succumbed to the framing effect, whereas approximately $29 \%$ of older adults succumbed. The raw data for this analysis are presented in Appendix L.

The second research question asked whether age, sex, or the interaction of age and sex predicted which group of participants was most likely to succumb to the framing effect. Logistic regression was used for this analysis. The overall model was not significant, $\chi^{2}(3, N=114)=2.85, p=.42$. This finding indicates that age and sex were not significant predictors of who exhibited the framing effect.

The final research question asked whether the relevance of the reasons listed on the debiasing questionnaire predicted whether participants exhibited the framing effect. 
These analyses were conducted separately for younger and older adults. The relevance of participants' reasons was scored according to a scoring rubric developed for the purpose of this study. The rubric outlined all the possible advantages and disadvantages of each treatment, as presented in the scenarios and the participant instructions. One point was given for each written answer that matched a possible answer on the scoring rubric. For example, a response of, "I've seen my friends go through radiation treatment and it didn't work for them" was scored a zero, whereas a response of, "Radiation treatment requires many trips to the hospital" was scored a one, since this information was presented in the participant instructions. These points were then summed for each participant to produce a total score for the debiasing questionnaires. This scoring procedure is based on prior debiasing studies (e.g., Miller \& Fagley, 1991; Sieck \& Yates, 1997; Mashat, 2004) which found that participants must consider information relevant to the scenario in order to eliminate the framing effect. The scoring rubric used is presented in Appendix M. Interrater reliability was computed for $20 \%$ of the data, which was selected at random. The intraclass correlation coefficient (ICC) was used as the measure of interrater reliability, due to the continuous variables produced by the scoring rubric. For the debiasing questionnaires, an ICC value of .982 was obtained. Logistic regression analyses revealed that for both the younger, $\chi 2(1, N=32)=.26, p=.61$, and older adult groups, $\chi 2(1, N=27)=1.43, p=.23$, the relevance of the reasons listed on the debiasing questionnaire did not predict whether participants succumbed to the framing effect. 


\section{Exploratory Analyses}

Several post-hoc analyses were conducted to further examine potential predictors that contributed to exhibiting the framing effect. The first exploratory analysis asked whether self-rated health status and variables related to the vicarious experience of cancer and decision making predicted whether participants succumbed to the framing effect. The questions about vicarious experience asked whether the participant had ever received or provided care to someone, whether they ever discussed the treatment of cancer with someone going through it, and whether someone close to them had ever been diagnosed with cancer. Logistic regression was used for these analyses and they were conducted separately for the younger and older adult groups. The criterion variable was whether the participant exhibited the framing effect.

For the younger adult group, the overall model was not significant, $\chi 2(6, N=61)$ $=6.69, p=.35$, indicating that neither self-rated health status nor the vicarious experience variables predicted whether participants exhibited the framing effect. There were significant results for the older adult group, which are presented in Table 6 . 
Table 6

Older Adult Health and Vicarious Experience Variables Predicting Framing Effect $(n=55)$

\begin{tabular}{lcccc}
\hline Variable & $B$ & $S E$ & Odds Ratio & Wald statistic \\
\hline Health & -4.64 & 31.37 & .01 & 8.06 \\
Poor & 2.38 & 1.90 & 10.76 & .02 \\
Fair & 4.51 & 2.03 & 91.11 & 4.56 \\
Average & 1.82 & 1.71 & 6.19 & 1.14 \\
Good & -2.41 & 1.15 & .09 & $4.41^{*}$ \\
Someone close diagnosed & -2.20 & .94 & .11 & $5.47 *$ \\
Care Receiver/Provider & -1.16 & .91 & .31 & 1.63 \\
Discussed Cancer & & & &
\end{tabular}

Older adults who knew someone close to them who was diagnosed with cancer (O.R., .09, $p<.05)$ were less likely to exhibit the framing effect than those who did not know someone diagnosed with cancer. Additionally, those who had received or provided care at some point in their lives (O.R., .11, $p<.05)$ were less likely to exhibit the framing effect than those who had never received or provided care.

The second exploratory analysis addressed the question of whether, for the control group, the number of relevant answers provided in the control questionnaires predicted the framing effect. The control questionnaires focused on what the participant would be willing to do to improve health in certain areas. Participants who were able to produce 
more potential ways to improve their health were potentially more health conscious, and therefore might be less likely to exhibit the framing effect.

A scoring rubric was developed for the control questionnaires, similar to the one developed for the debiasing questionnaires. Participants were given points for demonstrating willingness to change, enumerating what they would change, and acknowledging whether these changes would affect their day-to-day life. These point values were then summed to determine a total score for each participant for the control questionnaires. To determine interrater reliability, $20 \%$ of the data were dual-scored, which produced an intraclass correlation coefficient of .97. Logistic regression analyses revealed that for both younger, $\chi^{2}(1, N=32)=1.65, p=.20$, and older adults, $\chi^{2}(1, N=$ $29)=1.82, p=.18$, the number of relevant reasons provided on the control questionnaires did not predict whether participants succumbed to the framing effect. The scoring rubric is presented in Appendix M.

The third exploratory question asked whether the number of reasons provided for the question, "What pieces of information were the most important in making your decision and why?" differed by group and/or age. This was the last question on the debiasing questionnaire, and was also answered by the control group in the Rationale Request section. The number of reasons provided by the participant was averaged across all three scenarios to produce one score for each participant. Across age groups, those in the control group provided an average of $2.77(S D=1.81)$ reasons, whereas those in the debias group provided an average of $2.02(S D=1.41)$ reasons. This difference was statistically significant, $\mathrm{t}(121)=2.58, p=.01$. For the older adult group, there was no statistically significant difference between the number of reasons provided by the control 
$(M=1.72, S D=1.53)$ and debias $(M=1.13, S D=1.36)$ groups. For the younger adult group, the control group $(M=3.72, S D=1.51)$ provided significantly more reasons than the debiasing group $(M=2.90, S D=.75 ; \mathrm{t}(61)=2.71, p<.01)$. An examination of the differences between younger and older adults revealed that younger adults $(M=3.32, S D$ $=1.26)$ provided significantly more reasons than older adults $(M=1.36, S D=1.40$; $\mathrm{t}(119)=8.12, p<.001)$.

The final exploratory analysis determined which of three factors were most important in predicting treatment choice: age (young vs. old), frame (survival vs. mortality), or group (control vs. debias). Logistic regression analyses were used to examine the strength of these predictors for each of the three outcome information types. The criterion variable was whether the participant chose radiation or surgery. The results for the cumulative probability and interval probability formats are presented in Table 7 . 
Table 7

Prediction of Treatment Choice for Cumulative and Interval Probability Formats

\begin{tabular}{lcccc}
\hline Variable & $B$ & $S E$ & Odds Ratio & Wald Statistic \\
\hline Cumulative Probability $(\mathrm{n}=118)$ & & & & \\
Age & .27 & .39 & 1.32 & .51 \\
Frame & -1.11 & .39 & .33 & $8.37 *$ \\
Group & -.28 & .39 & .76 & .52 \\
Interval Probability $(\mathrm{n}=116)$ & -.22 & .40 & .80 & .30 \\
Age & -1.51 & .41 & .22 & $13.68^{* *}$ \\
Frame & .05 & .40 & 1.05 & .02 \\
Group & & & & \\
\hline$* p<.01, * * p<.001$ & & & & \\
\end{tabular}

Results indicated that for the cumulative probability format, frame was the only significant predictor; those in the survival frame were less likely to choose radiation than surgery (O.R., .33, $p<.01)$. There were similar results for the interval probability format. Frame was the only significant predictor, which indicated that those in the survival frame were less likely to choose radiation (O.R., .22, $p<.001$ ). There were no significant results for the overall life expectancy format, which also did not show a framing effect in the first and second hypotheses.

\section{Discussion}

Four hypotheses were tested in this study, and three were supported. The framing effect was demonstrated with younger and older adults, and the framing effect was precluded with younger adults using a debiasing procedure. The framing effect was not 
precluded with older adults. Results will be discussed separately for each hypothesis, followed by a discussion of three additional research questions and the results of four exploratory analyses.

\section{Demonstration of the Framing Effect with Younger Adults}

Hypothesis 1a proposed that the proportion of younger adults in the control group selecting surgery in the survival frame would differ significantly from the proportion selecting surgery in the mortality frame in at least one outcome information type, indicating an effect of frame. The younger adult control group exhibited the framing effect in the cumulative and interval probability formats, but not the life expectancy format. In the cumulative probability format, $68.8 \%$ of younger adults chose surgery in the survival frame, and the same percentage chose radiation in the mortality frame. In the interval probability format, $81.2 \%$ chose surgery in the survival frame, and $56.3 \%$ chose radiation in the mortality frame. This pattern of responses is similar to what has been shown in several studies on the framing effect in medical decision making using the same scenarios with younger adults (Tengs, 1987) and with younger adults, patients, and physicians (McNeil, Pauker, Sox, \& Tversky, 1987): surgery is chosen more with the survival frame, and radiation is chosen more with the mortality frame.

The results regarding the outcome information types that resulted in the framing effect differed slightly from the findings of other studies. For younger adult participants, Mashat (2004) found the framing effect only with the cumulative probability format, whereas McKee (2001) found a framing effect only with the life-expectancy outcome information format. Tengs (1987) found a framing effect in the life expectancy and cumulative probability formats, and McNeil et al. (1982) found a framing effect in only 
the life expectancy format, though this was across all participants in their study (students, patients, and physicians). Thus, there is little consistency between studies on what outcome information types produce the framing effect. McKee's (2001) findings could be partially due to the within-subjects design employed in her study. There is some consistency in producing the framing effect in the cumulative probability outcome type for younger adults when a between-groups design is employed.

\section{Demonstration of the Framing Effect with Older Adults}

Hypothesis $1 \mathrm{~b}$ proposed that the proportion of older adults in the control group selecting surgery in the survival frame would differ significantly from the proportion selecting surgery in the mortality frame in at least one outcome information type, indicating an effect of frame. The framing effect was obtained with only the interval probability format. In this format, $71.4 \%$ of older adults chose surgery in the survival frame, and the same percentage chose radiation in the mortality frame. Obtaining the framing effect only in the interval probability format is inconsistent with the findings of McKee (2001), who found a framing effect in only the life expectancy format for older adults. However, as mentioned above, this could be due to the design difference between her study and the current study, in that her study was a within-subjects design. The participants in McKee's (2001) study were making decisions for identical problems, which could have contributed to the "transparency" of the decision problem (Tversky \& Kahneman, 1986). Thus, participants may have noticed that they were being presented with discrepant information in each frame. Participants had an opportunity to compare scenarios in the within-subjects design, which participants could not do in the betweensubjects design. In McKee's (2001) study, the life expectancy condition could have 
produced a framing effect because comparison of the two scenarios would not reveal discrepant information, since the years of life expectancy for each treatment were the same in the survival and mortality conditions (e.g., 6.8 years for surgery, regardless of frame). However, in the other two outcome formats, the presented information was different depending on the frame of the problem.

Yates and Patalano (1999) offer a potential explanation for the presence of the framing effect in the interval probability format for older adults. They suggest that when presented with a cognitively demanding task, older adults are probably more likely to apply a rule-based decision-making approach that is faster and less cognitively demanding. Therefore, older adults may be more vulnerable to biases resulting from the use of rules or heuristics.

This account was supported by informal observations accumulated during testing sessions. Older adults often commented that the interval probability format was the most difficult to understand, which may have contributed to a higher reliance on heuristics and rule-based decision-making. Many participants read the interval probability scenario multiple times before making their treatment choice, which suggests that older adults may not have been able to retain the information in this outcome information type after one read through, or did not understand the information. In contrast, when the data were presented in terms of life expectancy, participants often stated that years of life post treatment was most important to them, suggesting that the decision appeared to be based solely on the life expectancy information. The life expectancy information was directly provided to the participant and therefore did not require any abstraction about the best choice based on the scenario. 
The lack of a framing effect with the cumulative probability format is difficult to explain. Since this format was presented first, the participants may have more carefully considered the details of the scenario, which could have had a debiasing effect. This level of consideration may have decreased with the increased cognitive complexity required for the interval probability outcome information, which was presented second.

\section{Debiasing Younger Adults}

Hypothesis 2a proposed that the proportion of younger adult participants in the debiasing group selecting surgery in the survival frame would not differ from the proportion selecting surgery in the mortality frame, indicating a successful avoidance of the framing effect. A framing effect was not present in the three outcome information types (cumulative probability, interval probability, and life expectancy). These results are consistent with those of Mashat (2004), who also demonstrated the debiasing in the cumulative probability format. However, Mashat failed to obtain a framing effect with the interval probability and life expectancy formats, which precluded a test of the debiasing with these formats. It is important to note that in the present study the debiasing procedure was not successful for everyone. If the debiasing procedure worked for everyone, there would have been an equal number of participants choosing each treatment within the survival and mortality frames. However, several participants chose surgery over radiation in the survival frame, or chose radiation over surgery in the mortality format. An examination of the data revealed that 14 younger adults showed the framing effect in the debiasing condition, indicating that the debiasing procedure did not work for these individuals. Nevertheless, the debiasing procedure appears to offer a 
potent remedy that can enable many, though not all, younger adults to avoid the consequences of biased decisions that are based in part on the framing of information.

\section{Debiasing Older Adults}

Hypothesis $2 \mathrm{~b}$ proposed that the proportion of older adult participants in the debiasing group selecting surgery in the survival frame would not differ from the proportion selecting surgery in the mortality frame, indicating a successful avoidance of the framing effect. The framing effect was obtained in the debias group with the cumulative and interval probability formats, but not with the overall life expectancy format. This result was unexpected. The older adults in the control group did not show a framing effect in the cumulative probability format, which is consistent with the results of McKee (2001), but participants in the debias group did show the framing effect. That is, when asked to consider the advantages and disadvantages of each treatment in the cumulative probability outcome type, older adults showed a framing effect, whereas the group that was asked to consider general information on stress did not show the effect.

The finding of a framing effect in the debiasing condition is difficult to explain since no framing effect was obtained without the debiasing instructions. These findings could be due to ineffective independent variable manipulation. For example, older adults potentially did not do what the instructions asked them to do. Another possibility is that the discrepant results are due to the design of the study, which produced two distinct control and debias groups. From a practical standpoint, these debiasing findings are not distressing, as there was no framing effect to preclude with the older adults in the control condition with the cumulative probability outcome information. 
The absence of a significant debiasing effect with the interval probability format is also puzzling. Careful consideration of information specific to the scenario appears to be the "active ingredient" in the debiasing procedure (Milley \& Fagley, 1991; Mashat, 2004). Informal observation of the older adult participants suggested that they were consistently not considering information presented in the scenarios. Instead, older adults often cited less relevant information gained from relatives or friends (e.g., "Radiation was very painful for my neighbor.”) If the cognitive demand of this outcome type was too high for the older adults' ability level, they may not have had the capacity to override the heuristic approach to decision-making and therefore may have relied more heavily on this approach, as evident in their reliance on less relevant personal information (cf., McElroy \& Seta, 2002). Older adults may also be relying on the availability heuristic when considering personally relevant information in their decision process. The information about their friends and relatives receiving cancer treatment may have been easier to recall from long-term memory, thereby influencing them to rely on this easily accessible information, instead of considering the information presented in the scenario (e.g., Hastie \& Dawes, 2001). The issue of older adults relying on more personally relevant information is discussed in the third exploratory analysis.

There are a few conclusions that can be drawn from the results of the main study hypotheses. First, younger adults appear susceptible to the framing effect within more framing conditions than older adults. The difference between younger and older adults across framing conditions is formally explored in a later section. This finding is inconsistent with what some authors (e.g., Park 1999) suggest should be the case. That is, older adults should be more likely to engage in an automatic processing approach, due to 
their experience with medical decisions and their decreased likelihood of engaging in controlled processing of decision-relevant information. Yates and Patalano (1999) have argued that older adults will use automatic processing whenever it can be applied to a scenario, which would decrease the likelihood that they would carefully consider all aspects of each problem, and increase the likelihood that they would succumb to the framing effect. It is also possible that different age groups employ different strategies for decision-making. Older adults may rely on more personally relevant information, but may not be relying on this personal information to the exclusion of other, more factually based, information.

In the current study, the framing effect manifested quite differently in older than younger adults. It was only present in what was arguably the most confusing format (interval probability format) with older adults, and the framing effect was still present in the control group with the interval probability format. This result presents a problem for those wishing to debias older adults with regard to the framing effect, specifically when information is presented in interval probability format. Participants in the present study worked through the decision materials on their own. Thus, there was no guarantee that the participants fully complied with the instructions. The debiasing procedure may have been more effective if each older adult participant was "walked through" the scenarios individually. A think-aloud procedure may have increased the likelihood that older adults complied with the instructions.

To summarize, three of the four main hypotheses were supported. The existing literature supports the presence of the framing effect with certain outcome information types, but the research is quite varied in terms of which outcome information types 
consistently produce the framing effect for younger and older adults. Why the present pattern of results was obtained is unclear from the available data. However, informal observation and speculation by other authors (e.g., Park, 1999; Yates \& Patalano, 1999; McElroy \& Seta, 2002) sheds some light on the reasons for the obtained results. Susceptibility to the Framing Effect

The first research question asked whether older or younger adults were more susceptible to the framing effect. Though no significant differences were found, a larger (statistically non-significant) percentage of younger adults succumbed to the framing effect. This result was surprising, since one might have expected the older adults to rely on more heuristic based reasoning to make their decision (see Park, 1999; Yates \& Patalano, 1999) when faced with substantial demands on working memory and cognitive capacity. Thus, they would have been expected to rely more on personal experiences and anecdotal evidence, rather than carefully considering the alternatives. If that were the case, one would have expected greater susceptibility to framing; that is, biased decision making.

As with the first research question, the second research question was an attempt to determine whether characteristics of the individual participants could explain some of the variance in predicting susceptibility to framing. The finding that neither age nor sex contributed to the prediction of framing was consistent with the findings regarding the first research question. Exploration of other individual difference variables (e.g., personality) may prove more fruitful, as there is evidence that impulsivity, anxiety, health involvement, and health negative affect correlate with attitudes that participants have towards risk-taking in a risky choice task (Lauriola, Russo, Lucidi, Violani, \& Levin, 
2005). However, these individual difference variables have yet to be demonstrated as predictors with the medical problems explored in the current study.

The third research question, which examined whether the relevance of reasons listed on the debiasing questionnaire could predict the framing effect, yielded nonsignificant findings. This question was based, in part, on work by Meyer et al. (1995), who found that older women offered less complete rationales for their decisions regarding treatment for breast cancer than younger women. The assumption in the present study was that if the older adults did not carefully consider the alternatives, they would offer fewer relevant reasons for their choices. Explanation of the results is difficult. The older adults may have carefully considered their choices, much as the younger adults, but may have had more trouble articulating why they made their selection. This could be due to older adults' heuristic based approach to the decision, whereas younger adults may be more able to articulate their reasons due to the use of a more analytic approach (e.g., McElroy \& Seta, 2002). However, this does not necessarily indicate that the older adults had no valid reasons for making their choices (Moye, Gurrera, Karel, Edelstein, \& O'Connell, 2006). A second possibility is that the coding scheme, which was developed for the present study, was inadequate for capturing differences in amount of information processing that occurred. For example, the current coding scheme was developed under the assumption that number of words written mattered less than the relevance of the reasons to the treatment choices. Therefore, a coding scheme that examined how completely participants were considering the alternatives (including relevant and seemingly irrelevant material) might have better captured the differences in information processing. 
The aim of the exploratory analyses was to examine whether individual difference variables predicted the framing effect. The present results suggest that prior personally acquired knowledge pertaining to cancer may inoculate one against the framing effect. However, it appears that this information needs to be relevant to the medical decision in order to have an inoculating effect. Older adult participants who had received or provided care at some point in their lives, and those who knew someone close to them who had been diagnosed with cancer, were less likely to exhibit the framing effect. This finding points to the importance of relevant information in the decision process. McElroy and Seta (2002) noted that in the area of medical decision-making, the less relevant the decision is to the participant, the more likely the framing effect will be exhibited. Therefore, this decision vignette may have been more relevant to those individuals who had been involved in these types of decisions in the past, thus inoculating them to the framing effect. In addition to the relevance of the decision vignette, individuals with relevant personal experiences may be considering more pieces of information before making their treatment choice. For example, Pierce (1996) found that individuals remembered the treatment choices that have been made by friends and family, and used that information to inform their own treatment choices. Additionally, Meyer et al. (1995) found that prior knowledge about a condition (breast cancer) influenced what treatment choice was selected. Therefore, in the present study, those older adult participants who knew someone close to them who had been diagnosed with cancer may have considered more information before making their decision, because they had been involved in similar decisions in the past. Likewise, participants who had received or provided care may have participated in medical decision making as a function of being a care provider. 
In addition, these individuals may have developed a network of individuals who had made a variety of medical decisions for themselves and their care recipients. Thus, there is a variety of research that supports the finding that those who have faced similar decisions in the past, therefore increasing the relevance of the current decision, may be less susceptible to the framing effect.

In examining predictors of the framing effect, we hypothesized that health conscious behavior may lead an individual to be less susceptible the effect of frame. Many of the questions on the control questionnaire tapped into health behaviors. For instance, participants were asked what they would be willing to do if their doctor told them they were suffering from stress, gum disease, or low levels of physical fitness. Therefore, we hypothesized that responses to these questions represented an individual's health conscious behaviors. Individuals with higher levels of health conscious behaviors were apparently no more likely to succumb to the framing effect than those with lower levels, as responses indicating willingness to change unhealthy behaviors on the control questionnaires failed to predict the framing effect.

\section{Consideration of Important Information in the Decision Process}

Across age groups, the control group provided more relevant reasons than the debias group when asked to answer the question, "What information is most important to you in making your decision?" This is counterintuitive to what would be expected. One might expect that since the debias group was specifically asked to consider the advantages and disadvantages of the treatments, they would provide more relevant reasons as to what influenced their final decision. It could be that the number of reasons is not important, but rather that individuals with only one reason had an important reason 
for considering only one piece of information. Additionally, the double presentation of the scenarios to the control group could account for the greater number of relevant reasons provided by the control group, as compared to the debias group. The control group was presented with the scenarios initially, and was then presented with them again for the Rationale Request section. Therefore they might have provided fewer reasons after the first presentation, if asked record their responses at that point.

The more interesting finding is the age differences in number of relevant reasons provided. The responses that older adults provided to this question were not as relevant to the information provided in the scenarios, and were based more on personal experiences. This finding is consistent with the results of Meyer et al. (1995) who found that older women offered less complete rationales for their treatment choice than younger women. However, it still has yet to be demonstrated that offering less complete rationales for decisions is related to lower quality decisions.

\section{Prediction of Treatment Choice}

The final exploratory analysis aimed to determine which variable was the best predictor of treatment choice: age (young vs. old), frame (survival vs. mortality), or group (control vs. debias). Previous studies (e.g., McNeil, Pauker, Sox. \& Tversky, 1982; Tengs, 1987; McKee, 2001) concluded that participants were more likely to choose surgery in the survival frame and radiation in the mortality frame. However, these studies collapsed across age groups in their sample, and therefore did not examine whether other variables, including age, were better predictors of treatment choice. The present results revealed that frame was the only significant predictor, out of frame, age, and group, for the cumulative and interval probability formats, with participants choosing 
surgery more often than radiation in the survival frame. This finding is consistent with the above studies, but McKee (2001) and McNeil et al (1982) did not rule out whether age was primarily responsible for the treatment decision.

\section{Limitations}

The results of the current study must be considered in light of some limitations. First, the present study considered treatments for only one medical condition (lung cancer). Although many participants drew on information from friends and family regarding treatments for other types of cancer, these results cannot be generalized to other types of cancer or to other acute and/or chronic medical conditions. Additionally, only two treatment choices were presented. Some older adult participants refused to pick one treatment, stating that they would probably have to end up getting both, or that they would choose neither. Therefore, the presentation of only two treatment options is a limitation, considering that many other treatment options, or combination of treatment options, are available to patients with lung cancer. Additionally, some participants may have had difficulty choosing between these two treatment options because they were unable to envision themselves ever facing these decisions. This could have been a barrier to producing accurate treatment decisions (as might be faced in real life) among the participants. Second, this study involved hypothetical scenarios. Although some of the older adult participants disclosed that they had gone through treatment for lung cancer, for many individuals the treatment decisions requested in this study were not decisions ever faced in their personal lives. This raises the possibility that individuals may make different decisions in the laboratory as compared to when they are faced with real life medical decisions, which are more relevant to their current situation. A related potential 
problem that can occur is the failure, or inability of older adults to place themselves in hypothetical situations. Third, the design of the study was cross-sectional, which only allows one to observe results exhibited by the two age groups, but does not allow one to conclude that age alone affects one's capacity to exhibit or avoid (through debiasing) the framing effect. Additionally, this type of design does not allow one to rule out cohort differences as part of the reason for differences among age groups. Finally, the sample was primarily Caucasian, and the older adult participants were primarily women. This sample reflects the population of rural Appalachia, but is not representative of all younger and older adults who face medical decisions. Therefore the findings may not be generalizable to other ethinicities, or even to a primarily older adult male sample.

\section{Conclusions}

The results of this study are further evidence that frame does play a role in treatment choice. The findings indicate that frame (survival vs. mortality), outcome information type (interval probabilities, cumulative probabilities, and overall life expectancy), and vicarious experience variables influence medical decision-making. Interestingly, the results on all the personal medical decision-making framing studies to date are opposite of what would be expected based on Prospect Theory (Kahneman \& Tversky, 1979). According to Prospect Theory, participants are expected to be risk averse when deciding between gains, and risk seeking when deciding between losses. Previous studies have identified surgery as the more risky treatment in the current medical scenarios (McNeil et al., 1982). Therefore, in medical scenarios, participants are consistently risk seeking when deciding between gains (choosing surgery in the survival 
frame), and risk averse when deciding between losses (choosing radiation in the mortality frame).

In the current study, older adults were less likely to succumb to the framing effect than younger adults, but the framing effect was not avoided by asking participants to consider the advantages and disadvantages of each treatment. There is inconclusive evidence in the current study and in the literature on the framing effect as to which outcome information types should consistently produce the framing effect. Comparison of results among studies is difficult, due to different study designs (between vs. withinsubjects), slight adaptations to the standard scenarios used in previous studies, and differences in administration of study materials. Although the current study did not produce a framing effect in the overall life expectancy format, other studies have found an effect with this outcome information type, which precludes us from drawing any conclusions as to the best way to present medical information. Further research which uses similar scenarios and a similar design is needed to produce clearer results regarding the conditions under which a framing effect is produced, and avoided, reliably.

The finding that experience with care and the discussion of cancer significantly reduces the likelihood that an older adult will exhibit the framing effect moves us one step closer to identifying individuals who are most susceptible to the framing effect, and offers a "natural" debiasing alternative. Additionally, for younger adults, the elimination of the framing effect may be as simple as asking them to consider the advantages and disadvantages of the treatments before making their treatment choice. Although it is alarming that a major medical decision can be influenced by something as simple as a 
change in the wording, it is equally important to note that there are simple and straightforward ways of eliminating this effect for certain populations.

\section{Future Directions}

Several future areas of research are suggested from the results of this study. First, the results of this study need to be replicated. The findings regarding which conditions produced a framing effect in younger and older adults were somewhat inconsistent with previous studies. Future studies need to ensure that the same scenarios are used and that the design of the study is similar, which will allow for easier comparison of results. Second, one might consider incorporating a friend or significant other into the decisionmaking process. In terms of ecological validity, a study that acknowledges the fact that many individuals discuss these types of decisions with friends or family members may produce different results (cf. Strough, Patrick, Swenson, Cheng, \& Barnes, 2003). Collaborative decision making could preclude a framing effect, due to the discussion that would be generated regarding both the advantages and disadvantages of the treatments and the incorporation of personal information. Additionally, the two age groups studied in the current study are very distinct. An examination into the framing effect among middle-aged and young-old adults is warranted, as these age groups might exhibit different patterns of choices than the participants in the present study. A longitudinal study would be preferable because it avoids any potential cohort effects and permits the researcher to pinpoint who is most susceptible to the framing effect, by examining susceptibility at various points throughout the lifespan. Although informal observations and results from the exploratory analyses from this study lends itself to the conclusion that older adults relied more on personally relevant information when making this 
specific medical decision, we still do not know exactly how older adults go about making medical decisions. An examination into this process is warranted. Finally, the use of situations in which older adults are facing medical decisions would strengthen the results of all of the framing effect studies to date, as this would eliminate the problem of the medical decisions not being relevant to the participants. This effect has been addressed through the use of hypothetical, laboratory based experiments, but has yet to be demonstrated in a real decision-making process. 


\section{References}

Arkes, H.R. (1981). Impediments to accurate clinical judgment and possible ways to minimize their impact. Journal of Consulting and Clinical Psychology, 49, 323-330.

Arkes, H.R., \& Blumer, C. (1985). The psychology of sunk cost. Organizational Behavior and Human Decision Processes, 35, 124-140.

Arkes, H.R., Faust, D., Guilmette, T.J., \& Hart, K. (1988). Eliminating the hindsight bias. Journal of Applied Psychology, 73, 305-307.

Arkes, H.R. (1991). Costs and benefits of judgment errors: Implications for debiasing. Psychological Bulletin, 110, 486-498.

Armstrong, K, Schwartz, J.S., Fitzgerald, G., Putt, M. \& Ubel, P.A. (2002). Effect of framing as gain versus loss on understanding and hypothetical treatment choices: Survival and mortality curves. Medical Decision Making, 22, 76-83.

Bernstein, L.M., Chapman, G.B., \& Elstein, A.S. (1999). Framing effects in choices between multioutcome life-expectancy lotteries. Medical Decision Making, 19, 324-338.

Cassel, C.K., Rudberg, M.A, \& Olshansky, S.J. (1992). The price of success: Healthcare in an aging society. Health Affairs, 11, 87-99.

Chapman, G.B., \& Johnson, E.J. (2002). Incorporating the irrelevant: Anchors in judgments of belief and value. In T. Gilovich, D. Griffin, \& D. Kahneman (Eds.), Heuristics and biases (pp. 120-138). New York: Cambridge University Press. 
Craik, F.I.M. (2000). Age-related changes in human memory. In D. Park \& N. Schwarz (Eds.), Cognitive Aging: A Primer (pp. 75-92). Philadelphia: Psychology Press.

Dixon, R.A., Hultsch, D.F., Simon, E.W., \& von Eye, A., (1984) Verbal ability and text structure effects on adult age differences in text recall. Journal of Verbal Learning and Verbal Behavior, 23, 569-578.

Fischhoff, B. (1975). Hindsight $\neq$ foresight: The effect of outcome knowledge on judgment under uncertainty. Journal of Experimental Psychology: Human Perception and Performance, 1, 288-299.

Fischhoff, B. (1977). Perceived informativeness of facts. Journal of Experimental Psychology: Human Perception and Performance, 3, 349-358.

Gilovich, T. (1981). Seeing the past in the present: The effect of associations to familiar events on judgments and decisions. Journal of Personality and Social Psychology, 40, 797-808.

Hastie, R., \& Dawes, R.M. (2001). Rational choice in an uncertain world. London: Sage Publications.

Kahneman, D., \& Tversky, A. (1984). Choices, values, and frames. American Psychologist, 39, 341-350.

Kahneman, D., \& Tversky, A. (1979). Prospect theory: An analysis of decision under risk. Econometrica, 47, 263-291.

Lauriola, M., Russo, P.M., Lucidi, F., Violani, C., \& Levin, I.P. (2005). The role of personality in positively and negatively framed risky health decisions. Personality and Individual Differences, 38, 45-59. 
Levin, I.P. (1987b). Associative effects of information framing. Bulletin of the Psychonomic Society, 25, 85-86.

Levin, I.P., Schneider, S.L., \& Gaeth, G.J. (1998). All frames are not created equal: A typology and critical analysis of framing effects. Organizational Behavior and Human Decision Processes, 76, 149-188.

Mashat, S. (2004) Framing effect debiasing in medical decision making. Unpublished Honors thesis, West Virginia University.

Mayhorn, C.B., Fisk, A.D., \& Whittle, J.D. (2002). Decisions, decisions: Analysis of age, cohort, and time of testing on framing of risky decision options. Human Factors, 44, 515-521.

McElroy, T., \& Seta, J.J. (2002). Framing effects: An analytic-holistic perspective. Journal of Experimental Social Psychology, 39, 610-617.

McKee, D.R. (2001). The effects of framing on younger and older adults' medical decision making. Unpublished doctoral dissertation, West Virginia University.

McNeil, B.J., Pauker, S.G., Sox, H.C., \& Tversky, A. (1982). On the elicitation of preferences for alternative therapies. The New England Journal of Medicine, $306,1259-1262$.

Meyer, B.J.F., Russo, C., \& Talbot, A. (1995). Discourse comprehension and problem solving: Decisions about the treatment of breast cancer by women across the life span. Psychology and Aging, 10, 84-103.

Meyerowitz, B.E., \& Chaiken, S. (1987). The effect of message framing on breast self-examination attitudes, intentions, and behavior. Journal of Personality and Social Psychology, 52, 500-510. 
Miller, P.M., \& Fagley, N.S. (1991). The effects of framing, problem variations, and providing rationale on choice. Personality and Social Psychology Bulletin, 17, 517-522.

Moye, J., Gurrera, R.J., Karel, M.J., Edelstein, B., \& O’Connell, C. (in press). Empirical advances in the assessment of the capacity to consent to medical treatment: Clinical implications and research needs. Clinical Psychology Review.

Moxey, A., O’Connell, D, McGettigan, T., \& Henry, D. (2003). Describing treatment effects to patients: How they are expressed makes a difference. Journal of General Internal Medicine, 18, 948-959.

Park, D.C., Smith, A.D., Lautenschlager, G., Earles, J., Frieske, D., Zwahr, M., \& Gaines, C. (1996). Mediators of long-term memory performance across the life span. Psychology and Aging, 11, 621-637.

Park, D.C. (1999). Aging and the controlled and automatic processing of medical information and medical intentions. In D.C. Park, R.W. Morrell, \& K. Shifren (Eds.), Processing of Medical Information in Aging Patients (pp. 3-22).

London: Lawrence Erlbaum Associates, Inc.

Pierce, P.F. (1996). When the patient chooses: A description of decision behavior. Human Factors, 38, 278-287.

Reimer, T., Mata, R., \& Stoecklin, M. (2004). The use of heuristics in persuasion: Deriving cues on source expertise from argument quality. Current Research in Social Psychology, 10, 69-84. 
Rönnlund, M., Karlsson, E., Laggnäs, E., Larsson, L. \& Lindström, T. (2005). Risky decision making across three arenas of choice: Are younger and older adults differently susceptible to framing effects? Journal of General Psychology, 132, 81-92.

Salthouse, T.A. (1993). Speed mediation of adult age differences in cognition. Developmental Psychology, 27, 763-776.

Schneider, W. \& Shiffrin, R.M. (1977). Controlled and automatic human information processing: 1. Detection, search, and attention. Psychological Review, 84, 1-66.

Sieck, W., \& Yates, J.F. (1997). Exposition effects on decision making: Choice and confidence in choice. Organizational Behavior and Human Decision Processes, 70, 207-219.

Strough, J., Patrick, J.H., Swenson, L.M., Suling, C., \& Barnes, K.A. (2003). Collaborative everyday problem solving: Interpersonal relationships and problem dimensions. International Journal of Aging and Human Development, 56, 43-66.

Tengs, T.O. (1987). The framing effect in medical decision making. Unpublished master's thesis, University of Massachusetts.

Thorburn, S., Harvey, S.M., \& Ryan, E.A. (2005). HIV prevention heuristics and condom use among African-Americans at risk for HIV. AIDS Care, 17, 335-345.

Turk, D.C., \& Salovey, P. (1986). Clinical information processing: Bias inoculation. In R.E. Ingram (Ed.), Information processing approaches to clinical psychology (pp. 305-323). San Diego: Academic Press.

Tversky, A., \& Kahneman, D. (1974). Judgment under uncertainty: Heuristics and biases. Science, 185, 1124-1131 
Tversky, A., \& Kahneman, D. (1981). The framing of decisions and the psychology of choice. Science, 185, 1124-1131.

Tversky, A., \& Kahneman, D. (1986). Rational choice and the framing of decisions. Journal of Business, 59, 251-278.

U.S. Administration on Aging. (2003). A profile of older Americans: 2003. [On-line]. Available: http://www.aoa.gov/prof/statistics/profile/2003/2 pf.asp.

Yates, J.F., \& Patalano, A.L. (1999). Decision making and aging. In D.C. Park, R.W. Morrell, \& K. Shifren (Eds.), Processing of Medical Information in Aging Patients (pp. 3-22). London: Lawrence Erlbaum Associates, Inc. 
Appendix A

Demographic Questionnaire

Age:

Sex: (circle one) Male

Female

Years of Education:

Marital Status: (circle one)

Single $\quad$ Married Separated Divorced Widowed

Ethnicity: (race)

Caucasian (White)

African American (Black)

Asian American

Hispanic

Pacific Islander

Native American (American Indian/Alaskan Native)

Have you ever been diagnosed with a chronic illness (e.g., heart disease, diabetes, arthritis)? Yes No

How long has it been since you were examined by a doctor?

Please rate your current health status: 1 . Poor

2. Fair

3. Average

4. Good

5. Excellent

Do you smoke? Yes No

If yes, how many years have you smoked?

What is your current occupation or the occupation you pursued for the majority of your adult life?

Has someone close to you ever been diagnosed with any type of cancer?

Yes No

If yes, what type of cancer?

Please indicate your relationship to this person: 
Have you ever received or provided care for someone? Yes No

If yes, were you the care receiver or provider? (circle one) Receiver Provider

How long were you the care receiver or provider?

Have you ever discussed the experience and/or treatment of cancer with someone who had cancer? Yes No

If so, please rate how involved you were in this person's life during their experience with cancer:

$$
\text { 1---------2-------------3------------4-----------5 }
$$

not at all involved

very involved 
Appendix B

Participant Instructions

The following pages contain specific information about cancer treatments at several Chicago area hospitals. Each hospital has its own doctors and policies regarding patient care, approaches to treatment, and different survival rates for the various types of treatment. For each hospital, please indicate whether you prefer surgery or radiation therapy. Below are general descriptions of the treatments.

Surgery for lung cancer involves an operation on the lungs. Most patients are in the hospital for two to three weeks and have some pain around their incisions; they spend a month or so recuperating at home. After that they generally feel fine.

Radiation therapy for lung cancer involves the use of radiation to kill the tumor and requires coming to the hospital about four times a week for six weeks. Each treatment takes a few minutes, and during the treatment patients lie on a table as if they were having an x-ray. During the course of treatment, some patients develop nausea and vomiting, but by the end of six weeks they generally feel fine.

Thus, after the initial six weeks, patients treated with either surgery or radiation therapy feel about the same.

Please answer the following questions in the order that they appear. Do not read ahead. Once you've answered a question, do not return to it. 
Appendix C

\section{Survival Worded Hospital Vignettes}

(Cumulative Probability Information)

\section{$\underline{\text { Hospital } 1}$}

Of 100 patients having radiation therapy, all patients live through the treatment, 77

patients live for more than one year, and 22 patients live for more than five years.

Of 100 patients having surgery, 90 patients live through treatment, 68 patients live for more than one year, and 34 patients live for more than five years. 
(Interval Probability Information)

\section{$\underline{\text { Hospital } 2}$}

Of 100 patients having surgery, 90 patients live until the end of treatment, 78 patients live through treatment but less than one year, 44 patients live for one to five years, and 10 patients live longer than five years.

Of 100 patients having radiation therapy, all patients live through the end of treatment, 77 live through the treatment but less than one year, 22 patients live for one to five years, and 5 patients live for more than five years. 
(Life Expectancy Information)

\section{$\underline{\text { Hospital } 3}$}

Of 100 patients having surgery, 90 percent of the patients live through treatment. The patients who survive surgery have an average life expectancy of 6.8 years.

Of 100 patients having radiation therapy, all patients live through treatment. The patients who survive radiation therapy have an average life expectancy of 4.7 years. 


\section{Appendix D}

\section{Mortality Worded Hospital Vignettes}

(Cumulative Probability Information)

\section{$\underline{\text { Hospital } 1}$}

Of 100 patients having surgery, 10 die during the treatment, 32 die by one year, and 66 die by 5 years.

Of 100 patients having radiation therapy, no patients die during treatment, 23 die by one year, and 78 die by 5 years. 
(Interval Probability Information)

\section{Hospital 2}

Of 100 patients having radiation therapy, no patients die by the end of treatment, 32 die in the time interval between treatment and one year, 55 die in the interval between one and five years, and 22 die sometime after five years.

Of 100 patients having surgery, 10 patients die by the end of treatment, 22 patients die in the interval between treatment and one year, 34 patients die in the interval between one and five years, and 34 patients die sometime after five years. 
(Life Expectancy Information)

\section{$\underline{\text { Hospital } 3}$}

Of 100 patients having radiation therapy, no patients die during treatment. The patients who survive treatment have an average life expectancy of 4.7 years.

Of 100 patients having surgery, 10 percent of the patients die during treatment. The patients who survive surgery have an average life expectancy of 6.8 years. 


\begin{abstract}
Appendix E
Debiasing Questionnaire

Please answer the following questions before choosing your treatment for Hospital 1:
\end{abstract}

Hospital 1: What are the advantages of surgery?

What are the disadvantages of surgery?

What are the advantages of radiation?

What are the disadvantages of radiation?

What pieces of information are the most important in making your decision and why? 
Appendix F

Control Questionnaires

Please answer the following questions before choosing your treatment for Hospital 1:

Stress is a common psychological state that also has negative effects on the body. Stress has its harmful effects by altering the levels of certain hormones in the body, resulting in a considerable array of disorders, such as increased blood pressure, heart disease, obesity, diabetes, asthma, and even brain cell death.

Imagine that your doctor tells you that your body is suffering from the effects of too much stress and asks you to answer the following questions:

1. What changes in your day-to-day life would you be willing to make to reduce stress?

2. How willing would you be to do daily exercises proven to reduce stress (e.g., meditation, physical exercise, etc.)?

3. To what extent would these changes in your daily routine affect your day-to-day life? 
Please answer the following questions before choosing your treatment for Hospital 2:

For many people, dental hygiene is a serious problem. Gum disease, one of the most common disorders of the mouth, is an inflammation of the gums that is caused by bacteria in a plaque, a sticky, colorless film that constantly forms on teeth. If not removed through daily brushing and flossing, plaque can build up and the bacteria may infect not only your gums and teeth, but also the gum tissue and bone that support the teeth. This can cause the teeth to become loose, fall out, or have to be removed by a dentist.

Imagine that you have been diagnosed with gum disease and asked to answer the following questions:

1. What changes in your daily routine would you be willing to make to alleviate the symptoms of the disease?

2. What changes in your diet would you be willing to make to alleviate the disease?

3. How severe would you rate the occurrence of this disease as compared to other potential physical disorders that may affect you? 
Please answer the following questions before choosing your treatment for Hospital 3:

Physical fitness is at the heart of prevention of many physical disorders. Physical fitness has been shown to improve not only physical functioning but also mental wellness. Unfortunately, most Americans are not at the level of fitness recommended by health professionals. The increasing number of overweight and obsess people in this country has led to increased rates of heart disease, stroke, diabetes, and other ailments.

Imagine that you have been advised by your doctor to increase your physical fitness and asked to answer the following questions:

1. What changes in your diet would you be willing to make to improve your physical fitness?

2. What changes in your lifestyle would you be willing to make to improve your physical fitness?

3. How would the above changes likely affect your day-to-day life? 
Appendix G

Treatment Choice Questionnaire

Which cancer treatment would you prefer? Circle one:

Surgery

Radiation Therapy 


\section{Appendix $\mathrm{H}$ \\ Answer Sheet (Survival Scenarios)}

$\underline{\text { Hospital } 1}$

Of 100 patients having radiation therapy, all patients live through the treatment, 77

patients live for more than one year, and 22 patients live for more than five years.

Of 100 patients having surgery, 90 patients live through treatment, 68 patients live for more than one year, and 34 patients live for more than five years.

For this scenario, you chose: Radiation Surgery 


\section{$\underline{\text { Hospital } 2}$}

Of 100 patients having surgery, 90 patients live until the end of treatment, 78 patients live through treatment but less than one year, 44 patients live for one to five years, and 10 patients live longer than five years.

Of 100 patients having radiation therapy, all patients live through the end of treatment, 77 live through the treatment but less than one year, 22 patients live for one to five years, and 5 patients live for more than five years.

For this scenario, you chose: Radiation Surgery 


\section{$\underline{\text { Hospital } 3}$}

Of 100 patients having surgery, 90 percent of the patients live through treatment. The patients who survive surgery have an average life expectancy of 6.8 years.

Of 100 patients having radiation therapy, all patients live through treatment. The patients who survive radiation therapy have an average life expectancy of 4.7 years.

For this scenario, you chose: Radiation Surgery 


\section{Answer Sheet (Mortality Scenarios)}

\section{$\underline{\text { Hospital } 1}$}

Of 100 patients having surgery, 10 die during the treatment, 32 die by one year, and 66 die by 5 years.

Of 100 patients having radiation therapy, no patients die during treatment, 23 die by one years, and 78 die by 5 years.

\section{For this scenario, you chose: Radiation Surgery}




\section{$\underline{\text { Hospital } 2}$}

Of 100 patients having radiation therapy, no patients die by the end of treatment, 32 die in the time interval between treatment and one year, 55 die in the interval between one and five years, and 22 die sometime after five years.

Of 100 patients having surgery, 10 patients die by the end of treatment, 22 patients die in the interval between treatment and one year, 34 patients die in the interval between one and five years, and 34 patients die sometime after five years.

For this scenario, you chose: Radiation Surgery 


\section{$\underline{\text { Hospital } 3}$}

Of 100 patients having radiation therapy, no patients die during treatment. The patients who survive treatment have an average life expectancy of 4.7 years.

Of 100 patients having surgery, 10 percent of the patients die during treatment. The patients who survive surgery have an average life expectancy of 6.8 years.

For this scenario, you chose: Radiation Surgery 
Appendix I

Rationale Request

1) For the Hospital 1 scenario, what pieces of information were the most important in making your decision and why?

2) For the Hospital 2 scenario, what pieces of information were the most important in making your decision and why? 
3) For the Hospital 3 scenario, what pieces of information were the most important in making your decision and why? 


\section{Appendix J}

IRB Cover Letter

To the participant:

The following questionnaires are part of a study entitled "Medical decision-making" that is being conducted as part of a Master's thesis, in partial fulfillment of the requirements for the Master's of Science degree in West Virginia University's Department of Psychology.

The study is being conducted to investigate possible factors involved in medical decisionmaking.

All your responses on this survey will be completed in an anonymous manner and will be kept strictly confidential.

You may choose not to respond to any question on the survey which you are not comfortable answering, although your cooperation is helpful.

Your participation in this study is strictly voluntary and you may withdraw from the experiment at any time.

Should you decide to withdraw from the study or refuse to answer any or all questions on this survey, it will have no effect on your academic record, job status, or community standing.

Sincerely, Erin Woodhead Barry Edelstein, $\mathrm{PhD}$ 
Appendix K

\section{CONSENT AND INFORMATION FORM}

\section{Medical decision-making}

\section{Introduction}

I, , have been asked to participate in this research study, which has been explained to me by Erin Woodhead. This research is being conducted by Erin Woodhead, and Barry Edelstein, $\mathrm{PhD}$, to fulfill the requirements for a Master's thesis in the Department of Psychology at West Virginia University, under the supervision of Dr. Barry Edelstein, PhD.

\section{Purposes of the Study}

The purpose of this study is to learn more about how people make medical decisions. Approximately 60 younger adult participants and 60 older adult participants are expected to participate in this study.

\section{Description of Procedure}

This study involves the completion of a set of forms that asks the participant to read a medical scenario, complete a questionnaire, and then make a selection between medical treatments. We will also be asking some participants additional questions about the medical treatment they have chosen. All participants will be asked to complete a brief demographic questionnaire. It will take approximately 35 minutes to complete all the forms in this study.

I have been asked to read a medical scenario, complete a questionnaire, and make a selection between medical treatments. I have also been asked to answer some additional

Submission date

Page 1 of 3

$\overline{\text { initials }} \overline{\text { date }}$ 


\section{Medical decision-making}

questions about my treatment selection, if requested, and to complete a demographic questionnaire. The medical treatment selection will ask me questions about three hypothetical medical scenarios and will take approximately 20 minutes to complete. The decision rationale will only be asked of some participants. This form will ask me to provide a rationale for the decisions I have made in the hypothetical medical scenarios. This will take approximately 10 minutes to complete. The demographic questionnaire will ask me to state my personal characteristics and will take about 5 minutes to complete. I have been told that I may see the above forms before signing this consent and that I do not have to answer all the questions if I decide to participate.

\section{Risks and Discomforts}

There are no known or expected risks from participating in this study

\section{Alternatives}

I understand that I do not have to participate in this study

\section{Benefits}

I understand that this study is not expected to be of direct benefit to me, but the knowledge gained may be of benefit to others. If I am a younger adult participant, I will receive extra credit in my class for participation, but there are other ways I may earn extra credit. If I am an older adult participant, my name will be recorded for an opportunity to win $\$ 100$.

\section{Contact Persons}

For more information about this research, I can contact Erin Woodhead, at 304-376-3338, or her supervisor, Dr. Barry Edelstein at 304-293-2001. For information regarding my rights as a research subject, I may contact the Office of Research Compliance at 304-2937073.

\section{Confidentiality}

I understand that any information about me obtained as a result of my participation in this research will be kept as confidential as legally possible. My record will be kept in a locked safe in the Department of Psychology at West Virginia University. I understand 


\section{Medical decision-making}

that my research records and test results, just like hospital records, may be subpoenaed by court order or may be inspected by the study sponsor, federal regulatory authorities (including the Food and Drug Administration), or the IRB without my additional consent. In any publications that results from this research, neither my name nor any information from which I might be identified will be published without my consent.

\section{Voluntary Participation}

Participation in this study is voluntary. I understand that I am free to withdraw my consent to participate in this study at any time and that such refusal to participate will not affect my class standing, grades, or job status. Refusal to participate or withdrawal will involve no penalty to me. I have been given the opportunity to ask questions about the research, and I have received answers concerning areas I did not understand.

Upon signing this form, I will receive a copy.

I willingly consent to participate in this research.

Signature of Subject

Signature of Investigator
Date

Date
Time

Time

Submission date

Page 3 of 3 


\section{Appendix L}

Raw data from Contingency Tables used to create Tables 1 to 5

Table 1: Younger Adult Control Group

$$
\begin{array}{ccc}
\text { Cumulative Probability } & \text { Interval Probability } & \text { Life Expectancy } \\
(\mathrm{n}=32) & (\mathrm{n}=32) & (\mathrm{n}=32)
\end{array}
$$

Frame Surgery Radiation Surgery Radiation Surgery Radiation

$\begin{array}{llllllll}\text { Survival } & 11 & 5 & 13 & 3 & 10 & 6\end{array}$

$\begin{array}{lllllll}\text { Mortality } & 5 & 11 & 7 & 9 & 9 & 7\end{array}$

Table 2: Older Adult Control Group
Cumulative Probability Interval Probability Life Expectancy$$
(\mathrm{n}=28) \quad(\mathrm{n}=28) \quad(\mathrm{n}=29)
$$

Frame Surgery Radiation Surgery Radiation Surgery Radiation

$\begin{array}{lllllll}\text { Survival } & 8 & 7 & 10 & 4 & 11 & 4\end{array}$

$\begin{array}{lllllll}\text { Mortality } & 7 & 6 & 4 & 10 & 7 & 7\end{array}$

Table 3: Younger Adult Debias Group

$\begin{array}{ccc}\text { Cumulative Probability } & \text { Interval Probability } & \text { Life Expectancy } \\ (\mathrm{n}=31) & (\mathrm{n}=31) & (\mathrm{n}=31)\end{array}$

Frame Surgery Radiation Surgery Radiation Surgery Radiation

$\begin{array}{lllllll}\text { Survival } & 8 & 7 & 10 & 5 & 12 & 3\end{array}$

$\begin{array}{llllllll}\text { Mortality } & 5 & 11 & 8 & 8 & 10 & 6\end{array}$


Table 4: Older Adult Debias Group

$$
\begin{array}{ccc}
\text { Cumulative Probability } & \text { Interval Probability } & \text { Life Expectancy } \\
(\mathrm{n}=29) & (\mathrm{n}=27) & (\mathrm{n}=27)
\end{array}
$$

Frame Surgery Radiation Surgery Radiation Surgery Radiation

$\begin{array}{lllllll}\text { Survival } & 10 & 4 & 11 & 3 & 11 & 3\end{array}$

$\begin{array}{lllllll}\text { Mortality } & 4 & 11 & 5 & 8 & 8 & 5\end{array}$

Table 5: Exhibition of Framing Effect by Age $(\mathrm{n}=117)$

Did Show Framing Did Not Show Framing

Younger Adults

Older Adults
28

16
34

39 
Appendix M

Scoring Rubric for the Debiasing and Control Questionnaires

\section{Debiasing Questionnaire Scoring Rubric:}

\section{Open-Ended Questions Scoring Template:}

1 point for each correct answer (may get more than 1 point for each question). No points for answers that don't directly reference material provided in the hospital scenario.

Analyses: 1) more points on debias questionnaire = less likely to succumb to framing effect? 2) Those filling out the debiasing questionnaire have more reasons for Question 5 than do those filling out the same questions for the control rationale request.

\section{Survival Debias Hospital 1:}

Question 1:

1 point answers: more patients live past 5 years, longer survival rate

Question 2:

1 point answers: only 90 live through surgery, less people live for more than 1 year than for radiation, in hospital for 2-3 weeks, pain around incisions, one month recuperation time

Question 3:

1 point answers: no patients die during radiation, more live for more than one year compared to surgery

Question 4:

1 point answers: less people live past 5 years, lots of trips to hospital, nausea/vomiting

Question 5:

1 point for each statement that directly addresses something in the Hospital 1 scenario 1 point if they make a general statement about life expectancy influencing their decision

\section{Survival Debias Hospital 2:}

Question 1:

1 point answers: more patients live past 5 years, longer survival rate, more patients live in interval between 1 and 5 years, more live through treatment but less than one year.

Question 2:

1 point answers: only 90 out of 100 live through surgery, less people live for more than 1 year than for radiation, in hospital for 2-3 weeks, pain around incisions, one month recuperation time 
Question 3:

1 point answers: all patients live through radiation

Question 4:

1 point answers: less live through treatment but less than one year, less live from one to five years, less live more than 5 years, lots of trips to hospital, nausea/vomiting

Question 5:

1 point for each statement that directly addresses something in the Hospital 2 scenario 1 point if they make a general statement about life expectancy influencing their decision

\section{Survival Debias Hospital 3:}

Question 1:

1 point answers: longer live expectancy for those who survive surgery (6.8 years vs. 4.7 years)

Question 2:

1 point answers: only 90 out of 100 live through surgery, in hospital for 2-3 weeks, pain around incisions, 1 month recuperation time

Question 3:

1 point answers: all patients live through radiation

Question 4:

1 point answers: shorter life expectancy for those who survive treatment, lots of trips to hospital, nausea/vomiting

Question 5:

1 point for each statement that directly addresses something in the Hospital 3 scenario

1 point if they make a general statement about life expectancy influencing their decision

\section{Mortality Debias Hospital 1:}

Question 1:

1 point answers: less die by 5 years

Question 2:

1 point answers: more die by one year, 10 die during treatment, in hospital for 2-3 weeks, pain around incisions, 1 month recuperation time

Question 3:

1 point answers: no patients die during radiation, less die by one year than surgery figures

Question 4:

1 point answers: more people die by 5 years, lots of trips to hospital, nausea/vomiting 
Question 5:

1 point for each statement that directly addresses something in the Hospital 1 scenario

1 point if they make a general statement about life expectancy influencing their decision

\section{Mortality Debias Hospital 2:}

Question 1:

1 point answers: less die in interval between treatment and one year, less die in interval between 1 and 5 years.

Question 2:

1 point answers: 10 die during surgery, more die sometime after 5 years, in hospital for 23 weeks, pain around incisions, 1 month recuperation time

Question 3:

1 point answers: no die during radiation, less die sometime after 5 years

Question 4:

1 point answers: less live through treatment but less than one year, less live from one to five years, less live more than 5 years, lots of trips to hospital, nausea/vomiting

Question 5:

1 point for each statement that directly addresses something in the Hospital 2 scenario 1 point if they make a general statement about life expectancy influencing their decision

\section{Mortality Debias Hospital 3:}

Question 1:

1 point answers: longer live expectancy for those who survive surgery (6.8 years vs. 4.7 years)

Question 2:

1 point answers: 10 percent die during surgery, in hospital for 2-3 weeks, pain around incisions, 1 month recuperation time

Question 3:

1 point answers: no patients die during radiation

Question 4:

1 point answers: shorter life expectancy for those who survive treatment, lots of trips to hospital, nausea/vomiting

Question 5:

1 point for each statement that directly addresses something in the Hospital 3 scenario

1 point if they make a general statement about life expectancy influencing their decision 


\section{Rational Request Scoring}

1 point for each statement in each question that directly references something stated in the corresponding hospital scenario, or in the answers provided above for each hospital. Also, award 1 point for anything referenced in the participant instructions (front page).

Control Questionnaire Scoring Rubric:

Open-Ended Scoring for Control Questionnaires:

$\underline{\text { Stress Questionnaire }}$

1. What changes?

1 point for each change they'd be willing to make only 1 point if they wrote, "I'd do whatever the doctor recommended."

2. How willing?

0 points - not willing 1 points - indicate that they're willing, but don't provide a qualifier, or indicate that they're willing to exercise in general (repeating the question) 2 points - indicate willingness and provide a qualifier of how willing (e.g., very) 3 points - detail how willing with specific changes they would make (e.g., specific ways they would exercise)

3. How much would it affect your day to day routine?

0 points - wouldn't affect it much

1 point - general statement that it would help out

If they give specific ways, assign 1 point for each way they think it would help out.

Gum Disease Questionnaire

1. What changes?

1 point for each change they'd be willing to make

only 1 point if they write that they would do what the doctor recommended

2. What changes in diet?

1 point for each diet change that they list

3. How severe would you rate gum disease, compared to other diseasest?

$1-$ not as severe

2 - same severity

3 - more severe 


\section{Physical Fitness Questionnaire}

1. What changes in diet?

1 point for each diet change they'd be willing to make

only 1 point if they write that they would do what the doctor recommended

2. What lifestyle changes?

1 point for each lifestyle change listed

same as above if they would defer to the doctor

3. How would it affect your day-to-day life?

0 points - not at all affected

1 point - general statement that it would help out

If they give specific ways, assign 1 point for each way they think it would help out. 